
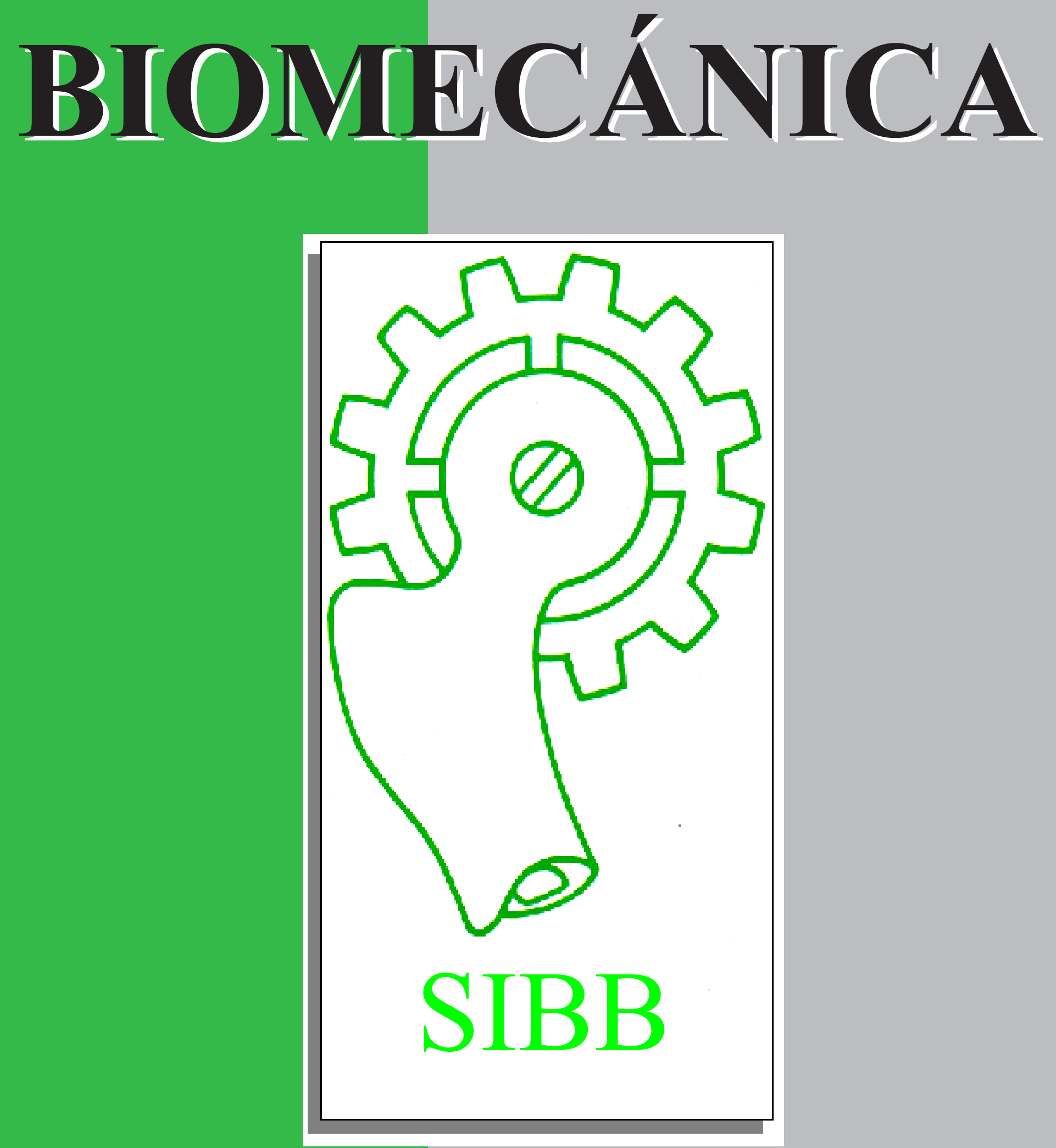

Órgano oficial de la Sociedad Ibérica de Biomecánica y Biomateriales

Volumen 26 2018

ISSN-e:1885-9518 


\section{BIOMECÁNICA}

Órgano Oficial de la Sociedad Ibérica de Biomecánica y Biomateriales

Página web SIBB: http://www.e-sibb.org

Página web Biomecánica: http://revistes.upc.edu/ojs/index.php/SIBB/

\section{Redacción:}

Diego Velasco Bayón / Daniel Rodríguez Rius

Dept. de Ciència dels Materials i Enginerya Metal·lúrgica

Escola d'Enginyeria de Barcelona Est (EEBE)

Universitat Politècnica de Catalunya (UPC)

Av. Eduard Maristany, 10-14, Edifici I

08930 - Sant Adrià del Besòs (Barcelona)

Correo electrónico: revista.biomecanica@e-sibb.org

\section{Especialidad:}

Especialistas en traumatología, medicina deportiva, rehabilitación, ingeniería biomecánica y biomateriales.

Periodicidad: 1 número al año

Depósito legal: B: 9920-2006

ISSN-e: $1885-9518$

ISSN: $1135-2205$ 


\section{BIOMECÁNICA}

\section{CONSEJO DE DIRECCIÓN}

Director - Diego Velasco Bayón

Director Adjunto - Daniel Rodríguez Rius

Secretario de Redacción - Pere Puig Mir

\section{COMITÉ CIENTÍFICO}

\section{Àrea de Cirugía Ortopédica y Traumatológica}

Coordinador - Joan Valentí Ardanuy

M. Fernández Fairén

J. Usabiaga Zarranz

A. Carranza Bencano

L. Gómez Pellico

\section{Àrea de Biomateriales}

Coordinador - Francisco Javier Gil Mur

E. Jorge Herrero

J. San Román del Barrio

J. J. Vázquez Solsona

F. J. Monteiro

M. Barbosa

\section{Àrea de Biomecánica Deportiva}

Coordinador - Marcos Gutiérrez Dávila
A. García Fojeda
K. Gianikellis
R. Arellano Colomina
P. Vera Luna

\section{JUNTA DIRECTIVA DE LA SIBB}

Presidente - Daniel Rodríguez Rius

Presidente electo - Enrique Navarro

Presidente saliente - José Antonio de Pedro Moro †

Secretario - Luis M. Rodríguez-Lorenzo

Tesorero - Esteban Colell

Director de la revista - Diego Velasco Bayón

Coordinadora página web - Maria Pau Ginebra Molins

Vocales

Pilar Bel Rafecas

José Campos Granell

Francisco Javier Gil Mur

Julio San Román del Barrio

Adoración Villarroya Aparicio

Antonio Viladot Voegli

\section{Antiguos Presidentes}

José Antonio de Pedro Moro † (2015-2016)

Francisco Javier Rojas Ruiz (2013-2015)

Juan Carlos Rodríguez Cabello (2011-2013)

Antonio Viladot Voegli (2009-2011)

José Campos Granell (2008-2009)

Julio San Román del Barrio (2005-2008)

Joan Valentí Ardanuy (2003-2005)

Marcos Gutierrez Dávila (2001-2003)

Francisco Javier Gil Mur (1999-2001)

Mariano Fernández-Fairén (1997-99)

Francisco Biosca Estela (1995-97)

Juan José Vázquez Solsona (1993-95)

Luís Fernando Llanos Alcázar (1991-93)

José Antonio Planell Estany (1987-90)

Luís García Font $\uparrow$ (1986)

Antonio Viladot Pericé † (1983-85)

Justo Nieto Nieto (1980-82)

Claudio Hernández Ros (1977-79)

\author{
Antiguos Directores de la Revista \\ Luis Fernando Llanos \\ Maria Pau Ginebra Molins \\ Josep Antón Planell Francisco Forriol \\ José Carlos Rodríguez Cabello \\ Conrado Aparicio Bádenas \\ Daniel Rodríguez Rius
}




\section{Normas editoriales}

\section{Información general}

Biomecánica publica trabajos originales, estudios de investigación, temas de actualización y colaboraciones especiales del ámbito de la traumatología, medicina deportiva, rehabilitación, ingeniería biomecánica y biomateriales:

\section{Correo electrónico: revista.biomecanica@e-sibb.org}

Correo postal:

Daniel Rodríguez Rius / Diego Velasco Bayón

Dept. de Ciència dels Materials i Enginerya Metal·lúrgica

Escola d'Enginyeria de Barcelona Est (EEBE)

Universitat Politècnica de Catalunya (UPC)

Av. Eduard Maristany, 10-14, Edifici I

08930 - Sant Adrià del Besòs (Barcelona)

Los manuscritos deben ser originales y no estar pendientes a publicarse en otras revistas. Biomecánica no se hace responsable de la opinión o criterio de los autores. Se reserva el derecho de propiedad de los trabajos publicados, que no deben ser reproducidos sin su autorización.

\section{Preparación de manuscritos.}

General: Los manuscritos deben ser entregados en procesador de texto (preferentemente en Word), a doble espacio con márgenes amplios y tamaño de fuente entre los 10 y 12 puntos. Conviene evitar un uso excesivo de formatos ya que la mayoría de los ellos serán eliminados o remplazados durante el procesamiento de su artículo. No utilice formatos automáticos de párrafo, tales como: justificación de texto, separación silábica, doble columna o enumeración de párrafos (especialmente para referencias numeradas). Sin embargo, se pueden utilizar formatos para las fuentes tales como negritas, cursivas, subíndices, superíndices, etc. Los editores se reservan el derecho de ajustar estilos con el fin de uniformar el formato. En la dirección de correspondencia debe especificarse claramente el destinatario, el número de fax y correo electrónico. También deberá anexarse la dirección postal completa de cada coautor. El estilo que se prefiere es: Apellido, Iniciales de Nombre, Departamento, Institución, Ciudad/ Comunidad, Código Postal, País. Los autores deberán conservar copias de todas las versiones del manuscrito enviadas a la revista.

Resumen: Todos los manuscritos deben entregarse con un resumen de entre 100 y 200 palabras.

Palabras clave: Los autores deben proporcionar entre 4 y 6 palabras clave para propósitos de índice.

Tanto el resumen como las palabras clave deben enviarse en inglés y español o portugués.

Texto: Siga este orden al construir su manuscrito: Título, Autores, Afiliaciones, Resumen, Texto, Agradecimientos, Apéndice, Bibliografía, leyendas de las Figuras y después Tablas. Las Figuras y Tablas deben presentarse en hojas aparte. No importe o inserte sus Figuras o Tablas en el texto. El autor a quien debe dirigirse la correspondencia debe identificarse con un asterisco y una nota al pie de página.

Unidades: Para todos los datos científicos y de laboratorio presentados deberá usarse el Sistema Internacional.

Introducción: Deben presentarse brevemente los antecedentes y el estado presente del tema de estudio.

Materiales y Métodos: Deben detallarse los materiales, protocolos experimentales, técnicas usadas, métodos de evaluación, etc. Los modelos experimentales deben describirse en suficiente detalle para permitir a otros investigadores la reproducción de los resultados. Debe incluirse una completa referencia al tipo de análisis estadístico empleado.

Resultados: Su presentación debe ser clara y concisa. Se recomienda el empleo de tablas y figuras. Todos los resultados expuestos deben haber sido obtenidos de acuerdo a la metodología descrita en la sección de Materiales y Métodos.
Discusión: Los resultados obtenidos e información que los respalde deben explicarse y correlacionarse con aquellos reportados en la literatura. En este sentido, es necesaria una revisión bibliográfica adecuada. Debe de subrayarse la importancia del estudio presentado y su proyección de futuro.

Conclusión: Deberá ser breve, haciendo énfasis en los aspectos originales más relevantes del estudio presentado.

Agradecimientos: Si fuesen necesarios, aparecerán después de las Conclusiones

Bibliografía: Todas las publicaciones citadas en el texto deben presentarse en una lista de referencias bibliográficas después del mismo en el manuscrito. En el texto, para referirse a la bibliografía, indíquela con un número consecutivo entre corchetes según orden de aparición (Ej. Ya que Peterson[1]), y una referencia completa debe de agregarse en la lista enumerada al final del escrito. La bibliografía debe seguir el siguiente formato:

1. Gough JE, Christian P, Scotchford CA, Rudd CD, Jones JA. Synthesis, degradation and in vitro cell responses of sodium phosphate glasses for craniofacial bone repair. J Biomed Mater Res 2002;59:481489 .

2. Nancollas H. In vitro studies of calcium phosphate crystallisation. In: Mann S, Webb J, Williams RJP, editors. Biomineralization. Chemical and biochemical perspectives. New York: VCH, 1989. p. 157-182.

3. Brown W, Chow LC. Combinations of sparingly soluble calcium phosphates in slurries and paste as mineralizers and cements. US Patent No. 4612053, 1986.

Tablas y figuras: Todas las tablas y figuras deben enumerarse independientemente, con numeración arábiga.

Tablas: no deben duplicar los resultados presentados en algún otro lugar dentro del manuscrito (por ejemplo en figuras). Debe proporcionarse un título adecuado para cada tabla. Éste debe escribirse antes de la tabla. No debe utilizarse reglas verticales, y conviene evitar tablas excesivamente complejas.

Figuras: Es necesario presentar versiones electrónicas de las ilustraciones, que pueden ser enviadas en archivos .jpg o importadas a Word o PowerPoint. Las leyendas de las figuras deben proporcionarse en una página independiente, después de la bibliografía. En microfotografías debe aparecer una barra de escala.

\section{Proceso editorial}

Los manuscritos serán evaluados por revisores independientes seleccionados por el Consejo de Redacción y el Comité Científico de Biomecánica. En caso de que el manuscrito necesite correcciones, estas tendrán que enviarse a la revista en un periodo no mayor a dos meses después de haber sido notificadas al autor. Una vez este tiempo haya concluido, si no hay respuesta, el manuscrito se devolverá al autor y se descartará su publicación. Ningún manuscrito será aceptado hasta que no se hayan hecho las correcciones correspondientes.

\section{Autorización de publicación}

Los artículos publicados en Biomecánica se distribuirán también electrónicamente mediante su incorporación en un repositorio electrónico.

Estos artículos se publican a través de una licencia de uso Creative Commons que permita a los usuarios de la revista su reproducción, distribución y comunicación pública siempre y cuando no se modifique la obra original, no se haga para un uso comercial y se cite la fuente original.

La publicación de los artículos en esta revista implica la aceptación por parte de los autores de las condiciones expresadas.

En ningún caso esta aceptación implica una cesión en exclusiva de los derechos de explotación del autor sobre la obra publicada ni impide 


\section{Instructions to authors}

\section{General information}

Biomecánica publishes original works, research studies, research updates and special collaborations in the fields of orthopedics, sports medicine, rehabilitation, biomechanics and biomaterials engineering. Manuscripts must be sent to the following address:

E-mail: revista.biomecanica@e-sibb.org

\section{Post mail:}

Daniel Rodríguez Rius / Diego Velasco Bayón

Dept. de Ciència dels Materials i Enginerya Metal·lúrgica

Escola d'Enginyeria de Barcelona Est (EEBE)

Universitat Politècnica de Catalunya (UPC)

Av. Eduard Maristany, 10-14, Edifici I

08930 - Sant Adrià del Besòs (Barcelona)

The manuscripts must be original, and should not be unde consideration for publication elsewhere. Biomecánica does not take any responsibility on the opinions and criteria of the authors, and it reserves the right of property of the works published, which should not be reproduced without its authorization.

\section{Manuscript preparation}

General: Manuscripts must be word processed (preferably in Word format), double-spaced with wide margins and a font size of 12 or 10 pt. Please avoid excessive layout styling as most formatting codes will be removed or replaced during the processing of your article. In addition, do not use options such as automatic word breaking, justified layout, double columns or automatic paragraph numbering (especially for numbered references). However do use bold face, italic, subscripts, superscripts etc. The corresponding author should be identified (include a Fax number and E-mail address). Full postal addresses must be given for all co-authors. The Editors reserve the right to adjust style to certain standards of uniformity. The preferred style is: Surname, Initials, Department, Institution, City/State, Postal Code, Country. Authors should retain copies of all versions of their manuscript submitted to the journal

Abstract: All manuscripts are to be supplied with an Abstract of about 100-200 words in length.

Keywords: Authors must provide 4-6 keywords for indexing purposes

The abstract and the keywords must be sent in English and Spanish or Portuguese.

Text: Follow this order when typing manuscripts: Title, Authors, Affiliations, Abstract, Keywords, Main text, Acknowledgements, Appendix, References, Figure Captions and then Tables. Do not import the Figures or Tables into your text. The corresponding author should be identified with an asterisk and footnote.

Units: The SI system should be used for all scientific and laboratory data.

Introduction: The background and present state of the subject of study must be presented briefly.

Material and methods: The materials and experimental protocols must be detailed, techniques used, methods of evaluation, etc. The experimental models must be described in sufficient detail to allow other researchers to reproduce the results. A complete reference to the type of statistical analysis used must be presented.

Results: They must be presented in a clear and concise form. The use of tables and figures is recommended. All of the exposed results mus have been obtained according to the methodology described in the Material and Methods section.
Discussion: The obtained results and their reliability must be explained, and correlated with those reported in the literature. In this sense, a suitable bibliographical revision is mandatory. It must also be stressed the importance of the study and its future projection.

Conclusion: It must be brief, underlying the more significant original aspects of the presented study.

Acknowledgments: If necessary, they will appear after the Conclusions.

References: All publications cited in the text should be presented in a list of references following the text of the manuscript. "In the text refer to references by a consecutive number in square brackets (e.g. Since Peterson [1]), and the full reference should be given in a numerical list at the end of the paper. References should be written in the following form:

1. Gough JE, Christian P, Scotchford CA, Rudd CD, Jones JA. Synthesis, degradation and in vitro cell responses of sodium phosphate glasses for craniofacial bone repair. J Biomed Mater Res 2002;59:481489.

2. Nancollas H. In vitro studies of calcium phosphate crystallisation. In: Mann S, Webb J, Williams RJP, editors. Biomineralization. Chemical and biochemical perspectives. New York: VCH, 1989. p. 157-182.

3. Brown W, Chow LC. Combinations of sparingly soluble calcium phosphates in slurries and paste as mineralizers and cements. US Patent No. 4612053, 1986

Tables and figures: All tables and figures must be numbered with independent Arabic numeration.

Tables: they should not duplicate results presented elsewhere in the manuscript, (e.g. in graphs). A suitable caption for each table must be provided. Footnotes to tables should be typed below the table and should be referred to by superscript lowercase letters. No vertical rules should be used. Excessively complex tables must be avoided.

Figures: Authors are required to provide electronic versions of their illustrations, which can be submitted either as .jpg files, or imported to Word or Power Point files. Figure captions must be provided in a separate page after the references. In microphotographs a scale bar must be marked on the photograph.

\section{Publishing process}

The manuscripts will be evaluated by independent referees selected by the Editorial Board and the Scientific Committee of Biomecánica. In case the manuscript might need corrections, these will have to be sent to the journal in less than one month after first receiving notice. Once this time elapsed, if there is no response, the manuscript will be given back to the author and it will be discarded for publication. No manuscript will be accepted until the complete corrections are made.

\section{Publishing authorization}

Articles published in Biomecánica are also distributed electronically through incorporation into an electronic repository.

These articles are published through a Creative Commons license that allows journal users reproduction, distribution and public communication, provided that the original work is not altered, is not used for commercial purposes and the original source is acknowledged. The authors of an article accept these conditions by its publication in Biomecánica.

In any case this acceptance implies an exclusive transfer of the author copyright on the published article or prevents normal exploitation of the article through the usual ways. 


\section{BIOMECÁNICA}

Órgano de la Sociedad Ibérica de Biomecánica y Biomateriales

\section{SUMARIO}

Volumen 26 (2018)

\section{ORIGINALES}

$7 \quad$ Estudio de la frecuencia de ocurrencia de accidentes de tráfico mediante procesos estocásticos de Pascal-Pólya

D. Sánchez-Molina, S. García-Vilana, J. Velázquez-Ameijide, C. Arregui-Dalmases

18 Electrodeposición de recubrimientos de fosfato de calcio sobre titanio

E. Vidal, E. Rupérez, D. Rodríguez

SIBB

25 Carta del director saliente

26 Carta del director

27 XLII Congreso de la Sociedad Ibérica de Biomecánica y Biomateriales 


\title{
Estudio de la frecuencia de ocurrencia de accidentes de tráfico mediante procesos estocásticos de Pascal-Pólya
}

\author{
D. Sánchez-Molina ${ }^{1}$, S. García-Vilana ${ }^{1}$, J. Velázquez-Ameijide ${ }^{1}$, C. Arregui-Dalmases ${ }^{2}$ \\ ${ }^{1} U P C$, EEBE-GRABI, Barcelona \\ ${ }^{2}$ Centro Zaragoza, Pedrola
}

\begin{abstract}
Resumen
Objetivos: (1) Plantear un modelo estocástico adecuado para la frecuencia de accidentes, que permita analizar tanto la peligrosidad, como la exposición al riesgo. (2) Introducir los conceptos de macroaccidentalidad y microaccidentalidad.

Métodos: En este artículo se han usado la teoría de procesos estocásticos para estimar el número de accidentes y así como la frecuencia con la que se producen accidentes de tráfico de una red viaria amplia (macroaccidentalidad). Para ello, se han analizado diversas bases de datos oficiales de una región durante un período prolongado de casi una década.

Resultados: El principal hallazgo es que un proceso estocástico de Poisson mixto (mezcla probabilista de varios procesos de Poisson) ajusta adecuadamente el número de accidentes, dando lugar a una distribución binomial negativa para el número de accidentes en un periodo especifico. Se propone una interpretación estocástica explicativa de las razones por las cuales la distribución del número de accidentes es de ese tipo.
\end{abstract}

Palabras clave: Distribución binomial negativa, Proceso de Poisson, Accidentes de tráfico

\begin{abstract}
Objectives: (1) To propose a stochastic model suitable for the frequency of accidents, which allows to analyze both dangerousness and risk exposure, and (2) Introduce the concepts of aggregate collision analysis (collision macroanalysis) and specific collision rate (collision microanalysis).

Methods: In this article, stochastic process theory has been used to estimate the number of traffic collisions and how often traffic collisions occur on a wide road network (collision macroanalysis). To this end, different official databases from the same area have been analyzed over an extended period, covering nearly a decade.

Results: The main finding is that a mixed Poisson stochastic process (probabilistic mixture of various Poisson processes) adequately represents the number of traffic collisions, resulting in a Negative Binomial Distribution for the number of collisions in a specific period. An explanatory stochastic interpretation is proposed for explaining why the number of collisions follows such a distribution.
\end{abstract}

Keywords: Negative Binomial Distribution, Poisson Process, Traffic Collision

Correspondencia:

David Sánchez.

E-mail: david.sanchez-molina@upc.edu 


\section{Introducción}

A nivel mundial, cada año alrededor de 1,25 millones de personas fallecen en accidentes de tráfico y entre 20 y 50 millones más sufren traumatismos de cierta consideración [1]. En muchos casos estas lesiones derivan en una discapacidad adquirida. La misma fuente (OMS) destaca que los accidentes de tráfico son ya la principal causa de muerte en personas de entre 5 y 29 años a nivel mundial. No obstante, debe señalarse que las tasas de mortalidad varían mucho según la región: así, el número de fallecidos en accidente de tráfico por cada 100 mil habitantes es 26,6 en África, mientras que para Europa es sólo de 9,3 por cada 100 mil.

Más específicamente en el contexto europeo, se observa que en España en 2016 se produjeron 1,85 millones de accidentes de tráfico al año, de acuerdo con los datos oficiales registrados [2]. En ese mismo año, la cifra de fallecidos por accidente de tráfico fue 1160 y el número de heridos graves fue de 140 mil.

Las cifras anteriores muestran por sí mismas la importancia epidemiológica, social y económica que los accidentes de tráfico representan y la razón por la cual es importante estudiar las causas de la accidentalidad por regiones, mediante modelos estadísticos fiables que modelicen la frecuencia de ocurrencia de los accidentes de tráfico (macroaccidentalidad) y como se relaciona esta ocurrencia con las características peculiares de conductores, conductas al volante, tecnología de los vehículos, estados de la vía y situaciones particulares de puntos específicos de la red (microaccidentalidad).

En cuanto a la modelización de la frecuencia del número de accidentes se han intentado diversas metodologías. Algunos autores han encontrado el mismo resultado principal que aquí se expone: que el número previsto de accidentes en muchas situaciones puede venir representado por una distribución binomial negativa, la mayor parte de trabajos a partir de mediados de los años 1990 usan ese tipo de distribución para la modelización $[3,4]$. Otros estudios previos se habían basado en la distribución de Poisson [5,6], aunque esos modelos parecen haber sido abandonados en gran medida, ya que los estudios recientes con datos mejores encuentran evidencias en favor del uso de un modelo binomial negativo [7,8,9]. En estos últimos trabajos prácticamente la única razón aportada para el modelo binomial negativo es que proporciona un mejor ajuste, pero no se incide en una explicación teórica de por qué cabría esperar ese tipo de distribución, asunto que en este artículo trata de aclararse desde el punto de vista de la teoría de procesos estocásticos.

El artículo se organiza de la siguiente manera: en la sección Conceptos previos se aclaran las nociones de peligrosidad, exposición al riesgo, macroaccidentalidad y microaccidentalidad y en las secciones Procesos estocásticos de Poisson y distribuciones compuestas y Proceso de Poisson mixto y distribución binomial negativa se aporta el núcleo teórico del artículo donde se toma el punto de vista de los procesos estocásticos. La sección $\mathrm{Ba}$ ses de datos explica la estructura de la base de datos que se usó para ilustrar el análisis. En la sección Peligrosidades y Exposiciones al riesgo especificas se dan las peligrosidades y exposiciones al riesgo bajo diversas circunstancias donde se aprecia qué factores temporales afectan a la frecuencia, y en la sección Análisis de la evolución de la peligrosidad se analiza la variación de la peligrosidad según situaciones específicas. En la sección Discusión se discute la importancia del resultado y se discuten otros enfoques alternativos que si bien proporcionan buenos ajustes numéricos carecen de profundidad teórica, y se argumenta por qué los procesos estocásticos si tienen un valor explicativo a diferencia de otros enfoques.

\section{Datos y Métodos}

\section{Conceptos previos}

El número de accidentes varía en función de la época del año y los días de la semana, siendo los períodos vacacionales los de menor siniestralidad y los viernes los días de mayor accidentalidad. Estas variaciones particulares tienen que ver con la intensidad del tráfico que resulta ser menor en períodos vacacionales y 
fines de semana, por lo que cualquier análisis debe considerar esa variación estacional dentro del año y de la semana. En esta sección seguiremos la terminología establecida en [10].

Para representar la siniestralidad agregada, número de accidentes de cada tipo, se recurre a definir la peligrosidad. Esta peligrosidad es una medida numérica directamente relacionada con la probabilidad de ocurrencia de accidentes, por tanto, este parámetro tendrá un valor instantáneo más alto cuando más accidentes se producen; de hecho, una medida adecuada de la peligrosidad debe satisfacer dos condiciones:

1. Debe ser calculable a partir de la distribución del número de accidentes producidos bajo una situación concreta (año, mes, día de la semana, tipo de vehículo, etc.)

2. Debe dar una idea de la distribución de probabilidad de los accidentes cuando la propia peligrosidad aumenta o disminuye.

Por tanto, para construir una media de peligrosidad se necesita estudiar las distribuciones de probabilidad del número de accidentes totales, lo cual da una peligrosidad general $\pi_{0}$. La peligrosidad especifica $\pi_{e}$ es el resultado de modificar la peligrosidad general mediante modificadores de macroaccidentalidad $\varphi_{i}$, usando una relación del tipo:

$$
\pi_{e}=\pi_{0}\left(1-\varphi_{a}\right)\left(1-\varphi_{m}\right)\left(1-\varphi_{d}\right)\left(1-\varphi_{h}\right)
$$

donde $\varphi_{a}$ es un factor que modifica la peligrosidad general que modifica la peligrosidad según el año, $\varphi_{m}$ según el mes del año, $\varphi_{d}$ es la peligrosidad debida al día de la semana y $\varphi_{h}$ debida a la franja horaria. Calculando las peligrosidades específicas por meses, días y franjas horarias es posible determinar $\sin$ dificultad las $\varphi_{i}$. En este estudio usamos como medida de la peligrosidad el valor esperado condicionado a ciertas circunstancias del conjunto $\sum$ (mes, día, hora), es decir $\pi_{e}=\mathbb{E}\left(N_{T}\right)<\operatorname{var}\left(N_{t}\right)$

Las medidas de peligrosidad son interesantes, pero ocultan un factor importante que es la varianza del número de accidentes, por usar únicamente la peligrosidad de una situación concreta puede ser engañoso si no se considera que situaciones de igual o muy parecida peligrosidad pueden presentar varianzas muy diferentes y, por tanto, existe la posibilidad de que en un período concreto el número de accidentes observados se aleje mucho del número estimado sólo a partir de la peligrosidad. Por esa razón se introduce la noción de exposición al riesgo que aquí definiremos como una combinación lineal de peligrosidad y desviación estándar:

$$
\mathrm{ER}_{T}=\mathbb{E}\left(N_{t}\right)+\mathrm{a} \sigma\left(N_{t}\right)
$$

donde $\mathrm{N}_{t}$ es la variable aleatoria "número de accidentes", $\mathbb{E}\left(N_{t}\right)$ es el valor esperado del número de accidentes (que es función de la peligrosidad), $\sigma\left(N_{t}\right)$ es la desviación tipo del número de accidentes y a es un parámetro real arbitrario, aquí hemos considerado $a=1,65$.

Este enfoque concentrado en las cifras agregadas de accidentes de todo tipo en una red viaria amplia, se conoce como macroaccidentalidad donde se hace abstracción de circunstancias específicas de los accidentes en particular de las características de los conductores y los puntos de la vía, mientras que el ajuste de la peligrosidad específica ajustada según el punto de la vía, poniendo atención al tipo de maniobras, perfiles de conductores y situaciones idiosincráticas del accidente se conoce como microaccidentalidad (la terminología comparte algunas de las características que diferencian el estudio agregado de cuestiones económicas o macroeconomía y el estudio pormenorizado de las decisiones de los agentes económicos en situaciones específicas o microeconomía).

\section{Procesos estocásticos de Poisson y distribu- ciones compuestas}

La teoría de procesos estocásticos se ocupa de variables aleatorias que "evolucionan" en el tiempo. Dada una magnitud aleatoria dependiente del tiempo es conveniente considerar una sucesión continua de variables aleatorias sobre el mismo espacio de probabilidad 
$(\Omega, \mathscr{F}, \mathbb{P})$, es decir, consideramos un conjunto indexado por el tiempo $\mathscr{X}=\left\{X_{t} \mid t \geq 0, X_{t}: \Omega\right.$ $\rightarrow \mathbb{R}\}$. El número de accidentes $N_{t}$ producidos en el intervalo $(0, t]$ es un ejemplo de proceso estocástico, ya que para un tiempo $t$ dicho número es una variable aleatoria y además se cumple que con probabilidad uno, $N_{t}$ es una magnitud no-decreciente.

Existen muchos tipos de procesos estocásticos, en función de que los eventos representados sean independientes o no, se distribuyan uniformemente en el tiempo o no, etc. Tal vez el tipo más simple de proceso estocástico sea un proceso de contaje (por tanto, el resultado será siempre un número natural) y donde la aparición de un nuevo evento (o accidente) es independiente de la ocurrencia de los anteriores. Los accidentes de tráfico parecen ser de ese tipo, dado lo esporádico de los mismos la ocurrencia de un accidente en una vía un martes, no afecta significativamente a la probabilidad de que haya otro accidente en el mismo punto o uno cercano una semana después (es decir, podemos pensar que los accidentes no están causalmente relacionados entre sí, lo cual es un buen punto de partida). Si las condiciones fueran siempre homogéneas (clima, intensidad de tráfico, tipo de conductor, mismo día de la semana, etc.), sería razonable pensar que el número de accidentes viene dado por un proceso de Poisson, cuyo nombre se debe a que al estudiar un período amplio $T$ el número de accidentes se ajusta a una distribución de Poisson del tipo:

$p_{n}(T)=\mathbb{P}\left(N_{T}=n\right)=\ldots$

$\ldots=\mathbb{P}(\{\omega \in \Omega \mid N(T, \omega)=n\})=\frac{(\lambda T)^{n}}{n !} e^{-\lambda T}$

donde $\lambda$ resulta ser la probabilidad por unidad de tiempo de ocurrencia de un accidente, que puede identificarse con la peligrosidad general. Puede demostrarse que esta distribución surge de suponer que el número de accidentes $N_{t}$ viene definido por un proceso estocástico de Poisson $N_{t}=N(t, \omega)$ que se basa en las asunciones siguientes [11]:
1. El proceso empieza en cero $N_{0}=N(0, \omega)$ $=0$, para todo $\omega$

2. El proceso tiene incrementos independientes, es decir, para una sucesión cualquiera de instantes $0<t_{0}<t_{1}<\ldots<t_{n}$, el número [aleatorio] de eventos en cada intervalo $\left(t_{i-1}, t_{i}\right]$ es una variable aleatoria denotada como $V_{i}=N\left(t_{i-1}, t_{i}\right]$, resultando que para todo $1 \leq i \leq n$ estas variables son mutuamente independientes (desde un punto de vista probabilista).

3. Si se define $N_{t}:=N(0, t)$, entonces el límite $\lim _{\Delta t \rightarrow 0} \mathbb{P}\left[N_{t+\Delta t}-N_{t} \mid N_{\tau}, \tau<t\right] / \Delta t$ está bien definido y describe la probabilidad por unidad de tiempo de que se produzca un nuevo evento $\mathrm{d} \mathbb{P}_{t} / \mathrm{d} t=\lambda_{t}$

4. Con probabilidad uno, los caminos $(N(t, \omega))_{t>0}$ son continuos por la derecha y admiten límites por la izquierda para $t>0$, esta condición define un proceso càdlàg (continue à droite, limites à gauche).

El proceso se llama homogéneo en el tiempo si $\lambda_{t}=\lambda_{0}$, es decir, la tasa de aparición de nuevos eventos es constante. La figura 1 presenta un ejemplo de proceso de Poisson y su probabilidad asociada (Figura 1).

En los primeros tiempos de la investigación de la frecuencia se empleó este tipo de proceso ya que el correlato físico de las condiciones anteriores es bastante razonable para la producción de accidentes de tráfico (si suponemos una población homogénea de conductores y vehículos, circulando por una red sin variaciones notorias de las condiciones de tráfico). Sin embargo, muy rápidamente la evidencia empírica mostró que la distribución de Poisson dada por la ecuación (3) no representaba adecuadamente los datos, ya que según esa distribución el valor esperado $\mathbb{E}\left(N_{t}\right)$ y la varianza $\operatorname{var}\left(N_{t}\right)=\mathbb{E}\left(N_{t}^{2}\right)-\mathbb{E}\left(N_{t}\right)^{2}$ para una distribución así coinciden $\mathbb{E}\left(N_{t}\right)=\operatorname{var}\left(N_{t}\right)=\lambda$ mientras que los datos experimentales mostraban una sobredispersión respecto a lo esperado, es decir, $\mathbb{E}\left(N_{t}\right)<\operatorname{var}\left(N_{t}\right)$. Eso es lo que llevó a buscar otro tipo de distribuciones que ajustaran mejor los datos. Por ejemplo, es conocido que 
una distribución de probabilidad compuesta donde se usa una distribución de cierto tipo, pero donde los parámetros que la definen se consideran en lugar de fijos distribuidos aleatoriamente puede resolver el problema de ajustar mejor unos datos que presenten sobredispersión respecto a la distribución en la que se basa el modelo. Es decir, si se tiene una variable aleatoria $X$ distribuida de acuerdo a cierta distribución $X \sim F_{\theta}$ donde los parámetros $\theta$ que caracterizan dicha distribución se asume que están distribuidos aleatoriamente $\theta \sim G$ entonces la distribución compuesta por $F$ y $G$ dará lugar a una distribución dada por:

$$
p_{F^{*} G}(x)=\int p_{F}(x \mid \theta) p_{G}(\theta) \mathrm{d} \theta
$$

La distribución resultante, para distribuciones $G$ suficientemente concentradas, será similar a la distribución $F$ pero tendrá sobredispersión ya que la varianza de la nueva distribución será:

$$
\begin{gathered}
\operatorname{var}_{F^{*} G}(X)=\mathbb{E}_{G}\left(\operatorname{var}_{F}(X \mid \theta)\right)+\ldots \\
\ldots \quad+\operatorname{var}_{G}\left(\mathbb{E}_{F}(X \mid \theta)\right)>\operatorname{var}_{F}(X \mid \theta)
\end{gathered}
$$

La mayor parte de estudios recientes sobre frecuencias de accidentes de tráfico han usado una distribución binomial negativa $[7,8,9]$, que corresponde a considerar una distribución auxiliar $G$ que sea una distribución gamma cuya densidad de probabilidad viene dada por:

$$
p_{G}(\theta)=\frac{\beta^{r}}{\Gamma(r)} \theta^{r-1} e^{-\beta \theta}
$$

$\mathrm{Y}$ algunos autores han llegado a usar una modificación conocida como distribución binomial negativa-Lindley [12,13] que es una generalización de la binomial negativa que pertenece a la familia Lindley de distribuciones descrita en [14]. Sin embargo, la mayor parte de estos trabajos simplemente pretenden salvar el problema de sobredispersión dar otra justificación de la distribución para la frecuencia de accidentes que el buen ajuste empírico a una distribución. En la siguiente sección se da una justificación teórica por la cual la distribución binomial negativa da un buen ajuste, a partir de la noción la noción de proceso de Poisson mixto.

A modo de resumen de esta sección se tiene que, aunque los procesos estocásticos de Poisson cualitativamente si dan una idea del

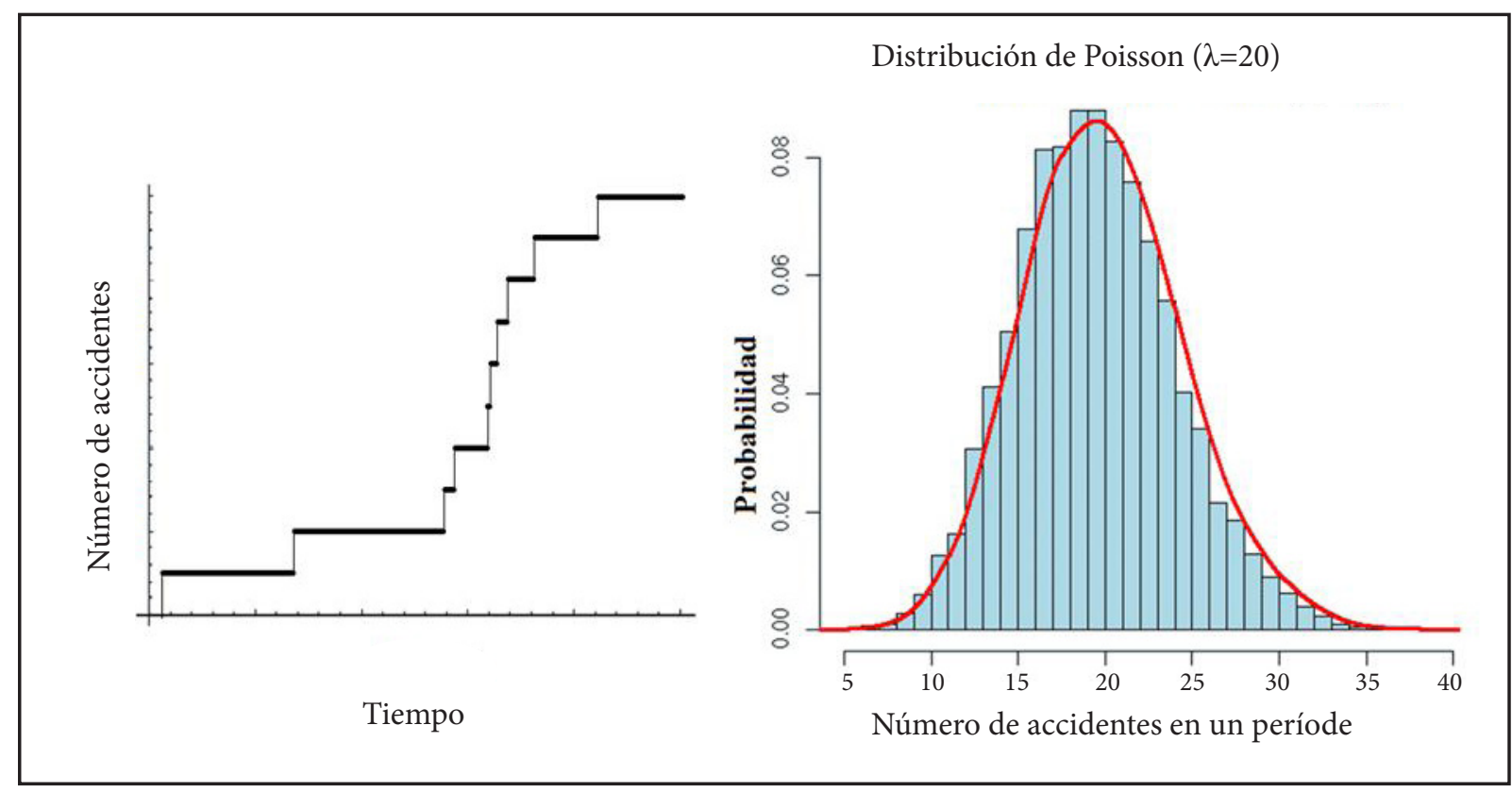

Figura 1. Izquierda: número de sucesos (accidentes) generados aleatoriamente por un proceso de Poisson homogéneo. Derecha: Distribución de probabilidad para un periodo fijo dada por un proceso de Poisson homogéneo. 
ritmo al que se producen accidentes, cuantitativamente, como se ha discutido anteriormente no reproducen bien la sobredispersión de los datos observados. Por otra parte, aunque las distribuciones compuestas como la binomial negativa reproducen bien la sobredispersión, la justificación teórica de la misma en la literatura ha sido insuficientemente tratada. En la siguiente sección se pretender resolver ambas deficiencias con una justificación teórica más sólida.

\section{Proceso de Poisson mixto y distribución bino-} mial negativa

Un proceso de Poisson mixto $\{\tilde{N}(t) \mid t>$ $0, \tilde{N}(t)=N(t, \omega) \in \mathbb{N}\}$ es un tipo de proceso estocástico más general donde la distribución de conteo viene dada por [15]:

$p_{n}(t)=\mathbb{P}\left(\left\{N_{T}=n\right\}\right)=\int_{0}^{\infty} \frac{(\lambda t)^{n}}{n !} e^{-\lambda t} \mathrm{~d} \mu(\lambda)$

donde $\mu(\lambda):=\mathbb{P}[0, \lambda]$ es la llamada distribución de estructura. Claramente, un proceso de Poisson homogéneo, es un caso particular de (7), para verlo basta considerar una medida $\mu(\lambda)$ expresable en términos de la delta de Dirac $\mathrm{d} \mu(\lambda)=\delta\left(\lambda-\lambda_{0}\right) \mathrm{d} \lambda$. Varios procesos estocásticos interesantes pueden ser representados de esta manera, para diferentes elecciones de la medida $\mathrm{d} \mu(\lambda)$, en particular escogiendo una medida asociada a la distribución gamma:

(8)

$$
\mathrm{d} \mu(\lambda)=\frac{\beta^{r}}{\Gamma(r)} \lambda^{r-1} e^{-\beta \lambda} \mathrm{d} \lambda
$$

que depende de dos parámetros $r \in \mathbb{N}$ y $\beta \in \mathbb{R}^{+}$. En este caso el proceso de Poisson mixto se puede entender como una mezcla de procesos de Poisson en los que cada uno tiene un peso diferente dado por $\mathrm{d} \mu(\lambda)$. Así, la accidentalidad en condiciones de lluvia es diferente, así como la accidentalidad en condiciones de poca visibilidad o la accidentabilidad de los conductores noveles respecto a los experimentados, el peso de cada tipo de condición es lo que la medida $\mathrm{d} \mu(\lambda)$ tiene en cuenta. Para la medida específica (8) la integral de la ecuación (7) puede llevarse a cabo de manera explícita resultando la forma cerrada de una binomial negativa:

$$
\begin{gathered}
\mathbb{P}\left(\left\{N_{T}=n\right\}\right)=\frac{\Gamma(r+n)}{\Gamma(r) n !}\left(\frac{t}{t+\beta}\right)^{n} \ldots \\
\ldots \quad\left(\frac{\beta}{t+\beta}\right)^{r}=\left(\begin{array}{c}
n+r-1 \\
n
\end{array}\right) p^{n}(1-p)^{r}
\end{gathered}
$$

donde el parámetro $p=t /(t+\beta)$. El proceso estocástico mixto dado por (7) y (8) se denomina proceso de Pascal [15] o proceso de Pólya [16,17].

Con el fin de hacer comparaciones con la distribución de Poisson se define el parámetro real $\rho=r / T$, donde $r$ es el parámetro entero que aparece en la distribución (8). En términos de este parámetro el número de accidentes durante un período $T$ viene dado por:

$$
p_{n}(T)=\frac{\Gamma(\rho T+n)}{\Gamma(r) n !} p^{n}(1-p)^{\rho T}
$$

Esta distribución vendrá dada por tanto por dos parámetros reales $0<p<1$ y $\rho$ que se pueden obtener mediante estimación de máxima verosimilitud a partir de los datos empíricos de la distribución de accidentes. Además, para la binomial negativa, el valor esperado y la varianza son:

$$
\begin{aligned}
\mathbb{E}\left(N_{T}\right) & =\frac{p}{1-p} \rho T \\
\operatorname{var}\left(N_{T}\right) & =\frac{p}{(1-p)^{2}} \rho T
\end{aligned}
$$

Obsérvese que ahora para el proceso de Pascal-Pólya se tiene $\operatorname{var}\left(N_{t}\right) / \mathbb{E}\left(N_{t}\right)>1$, mientras que para un proceso de Poisson se tenía $\operatorname{var}\left(N_{t}\right) / \mathbb{E}\left(N_{t}\right)=1$, lo cual permite dar cuenta de la sobredispersión observada en los datos.

Además, la peligrosidad y la exposición al riesgo estimadas por un proceso de Poisson, y un proceso de Pascal-Pólya pueden ser comparadas con facilidad. Si se compara la distribución binomial $\mathrm{BN}(p, \rho)$, dada por (10), con una distribución de Poisson $(\lambda)$, dada por (3), del mismo valor esperado se tiene la relación $p=\lambda /(\rho+\lambda)$, por lo que la tasa de accidentes por unidad de tiempo o peligrosidad de ambos procesos resultan ser iguales: 
(12)

$$
\pi_{\mathrm{Poi}}=\lambda=\frac{p}{1-p} \rho=\pi_{\mathrm{BN}}
$$

Para las exposiciones al riesgo, la sobredispersión del proceso de Pascal-Pólya da estimaciones algo mayores $\left(\mathrm{ER}_{\mathrm{BN}}>\mathrm{ER}_{\mathrm{Poi}}\right)$ :

$\mathrm{ER}_{\mathrm{Poi}}=\lambda\left(1+\frac{a}{\lambda}\right)=\frac{p \rho}{1-p}\left(1+\frac{a(1-p)}{\sqrt{p \rho}}\right) \ldots$

$$
\ldots<\frac{p \rho}{1-p}\left(1+\frac{a}{\sqrt{p \rho}}\right)=\mathrm{ER}_{\mathrm{BN}}
$$

\section{Bases de datos}

Para el estudio empírico se utilizó la base de datos SIDAT, mantenida por el Servei Català del Transit (SCT), organismo dependiente del gobierno autónomo de Cataluña. Esta es una base extensa que contiene 132 variables y está enfocada a la descripción exhaustiva de cada accidente registrado. Entre estas variables encontramos la localización del accidente, las características de la vía, la descripción de las unidades, además de la fecha, día y hora del accidente. También dependiendo de la gravedad del accidente se recogen detalles complementarios. En este estudio se consideraron todos los accidentes registrados entre 2007 y 2015 sobre la parte de la red viaria que es competencia del SCT. El número de registros encontrados en ese período es de 267 mil accidentes registrados, lo cual da más de 33 mil accidentes por año, que constituye una buena base para cualquier estudio estadístico.

\section{Resultados}

De los datos contenidos en la base de datos SIDAT, para el área de Cataluña se concluye que los meses más peligrosos son el período de mayo a julio y el período de noviembre a diciembre. El día más peligroso es el viernes y el de menos peligrosidad el domingo (esto claramente parece estar relacionado con el número de desplazamientos). En la muestra analizada, debido a la crisis económica española (2008-2014) se aprecia en la primera parte de ese período de 2008, 2009 y 2010 un número de accidente menor que la media del período 2007-2015. La franja horaria de entre las 11:00 y las 15:00 es la que concentra mayor número de accidentes.

\section{Peligrosidades y Exposiciones al riesgo especificas}

En esta sección se calcula la peligrosidad general por año y las peligrosidades específicas por mes y día de la semana, usando un modelo basado en procesos estocásticos. Como se hizo notar en la ecuación (12), las peligrosidades estimadas según un proceso de Poisson o un proceso de PascalPólya resultan ser idénticas, no así la exposición al riesgo que se muestra en las tablas. Esto se hizo comparando las frecuencias observadas con las distribuciones de Poisson y binomial negativa, en todos los casos se realizó un test $\chi^{2}$ para la bondad del ajuste. En general dicho test no fue superado por el proceso de Poisson, pero en todos los casos el test $\chi^{2}$ fue superado por el proceso de Pascal-Pólya que lleva a la binomial negativa. La figura 2 muestra un mejor ajuste estimado mediante el paquete estadís-

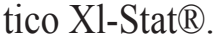

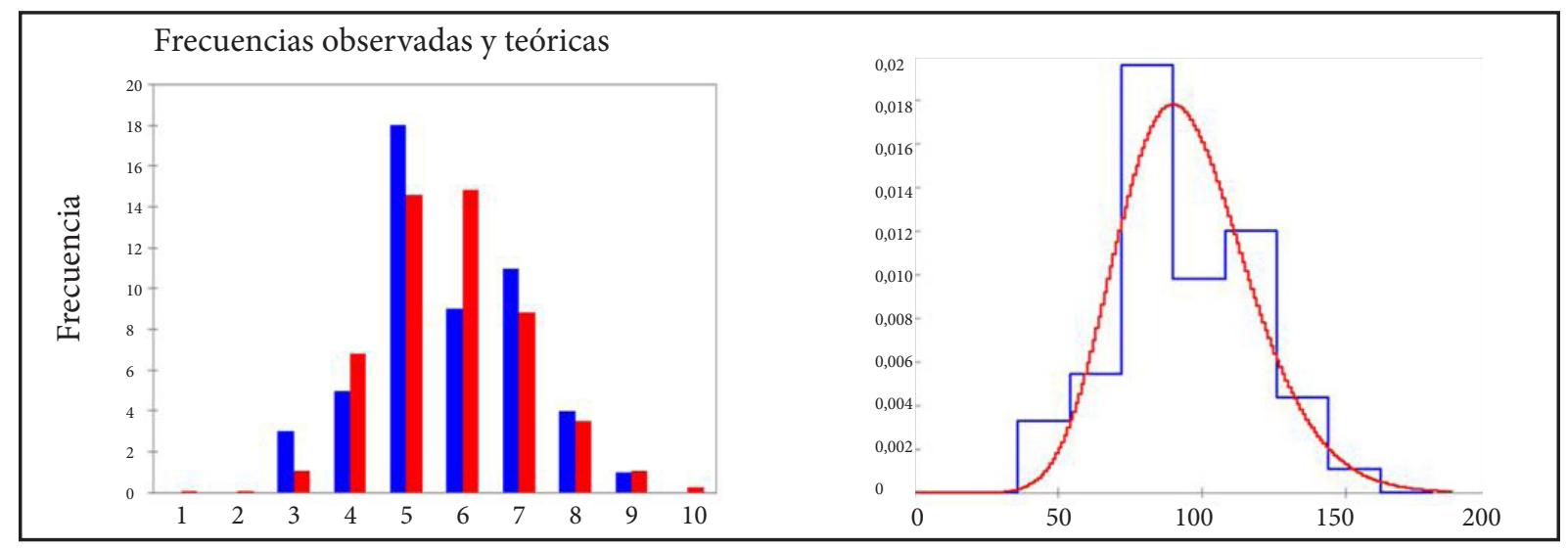

Figura 2. Izquierda: Número de accidentes observados y predichos por una binomial negativa. Derecha: Comparación entre observaciones y la envolvente de una binomial negativa. 
Las peligrosidades generales por año y por mes se dan en la tabla 1, mientras que las peligrosidades por día de la semana en la figura 3.

\section{Análisis de la evolución de la peligrosidad}

En esta sección se usan las peligrosidades generales y específicas de la sección anterior para entender cómo ha ido evolucionando la macroaccidentalidad general en Cataluña en el período (2007-2015). Esto se hace introduciendo los modificadores de macroaccidentalidad específicos $\varphi_{i}$ que se definen para cada

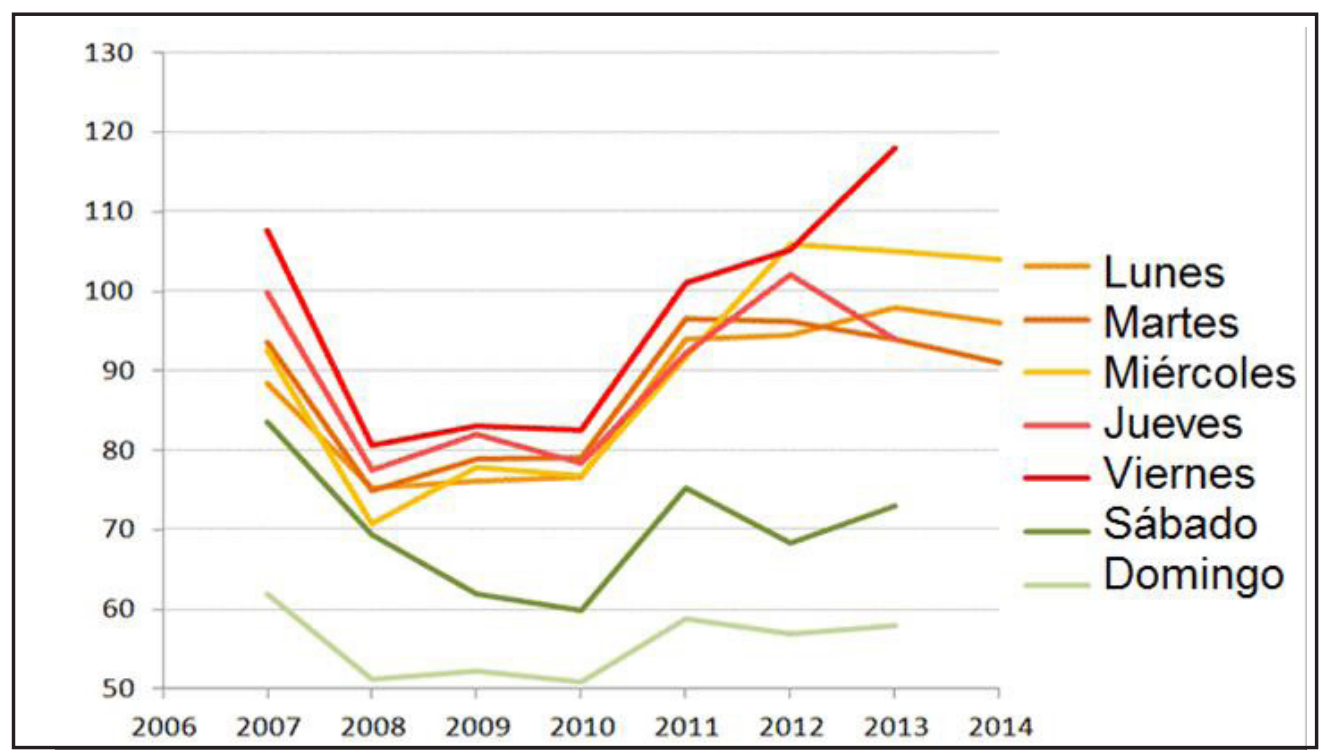

Figura 3. Peligrosidades calculadas para cada día de la semana, la figura muestra tan la mayor peligrosidad de los viernes, como la variación de la peligrosidad en años de mayor desempleo.

\begin{tabular}{|c|c|c|c|c|c|c|c|}
\hline & & & & \multicolumn{2}{|r|}{$\begin{array}{l}\text { Peligrosidad } \\
\text { específica }\end{array}$} & \multicolumn{2}{|c|}{$\begin{array}{c}\text { Exposición al riesgo } \\
\text { específica }\end{array}$} \\
\hline & & & & Mes & $\begin{array}{l}\text { Poisson y } \\
\text { Binomial } \\
\text { negativa }\end{array}$ & Poisson & $\begin{array}{l}\text { Binomial } \\
\text { negativa }\end{array}$ \\
\hline \multirow[b]{2}{*}{ Año } & \multirow{2}{*}{$\begin{array}{c}\begin{array}{c}\text { Peligrosidad } \\
\text { general }\end{array} \\
\text { Poisson y } \\
\text { Binomial negativa }\end{array}$} & \multicolumn{2}{|c|}{ Exposición al riesgo } & & 85,1 & 100,3 & 115,7 \\
\hline & & Poisson & $\begin{array}{c}\text { Binomial } \\
\text { negativa }\end{array}$ & $\begin{array}{l}\text { Febrero } \\
\text { Marzo }\end{array}$ & $\begin{array}{l}87,9 \\
84,1\end{array}$ & $\begin{array}{l}103,4 \\
99,2\end{array}$ & $\begin{array}{l}110,9 \\
103,9\end{array}$ \\
\hline 2007 & 87,1 & 93,0 & 104,8 & Abril & 81,7 & 96,6 & 117,8 \\
\hline 2008 & 71,7 & 76,9 & 83,8 & Mayo & 87,1 & 102,5 & 118,6 \\
\hline 2009 & 72 & 77,3 & 83,1 & Junio & 87,3 & 102,7 & 114,4 \\
\hline 2010 & 72,4 & 77,7 & 88,0 & & & & \\
\hline 2011 & 86,2 & 92,0 & 101,4 & Julio & 88,1 & 103,6 & 110,9 \\
\hline 2012 & 89,8 & 95,7 & 104,4 & Agosto & 69,1 & 82,8 & 107,4 \\
\hline 2013 & 90,5 & 96,5 & 108,9 & Sept. & 80,5 & 95,3 & 110,0 \\
\hline 2014 & 85,3 & 91,1 & 107,3 & Octubre & 85,5 & 100,8 & 111,0 \\
\hline 2015 & 88,0 & 93,8 & 110,4 & Noviem. & 86,5 & 101,8 & 118,1 \\
\hline Todos & 81,2 & 96,1 & 128,2 & Diciem. & 82,3 & 97,3 & 153,2 \\
\hline
\end{tabular}

Tabla 1. Peligrosidades calculadas para cada año y exposición al riesgo. Los años de mayor desempleo se registró un descenso del número de vehículos circulantes y del número de accidentes. 
año, cada mes y cada día en la forma sugerida por la ecuación (1). La ventaja de estos factores es que cuando son positivos indican que se esa situación presenta una peligrosidad específica mayor que la general y cuando son negativos indican una disminución del riesgo. Más específicamente usaremos los modificadores de macroaccidentalidad adoptados a la estructura de los datos, más específicamente cumplirán las siguientes condiciones:

(14) $\left\{\begin{array}{l}\pi_{a}=\pi_{0}\left(1-\varphi_{a}\right) \\ \pi_{m}=\pi_{0}\left(1-\varphi_{a}\right)\left(1-\varphi_{a m}\right) \\ \pi_{d}=\pi_{0}\left(1-\varphi_{a}\right)\left(1-\varphi_{a d}\right)\end{array}\right.$
Donde $\pi_{a}, \pi_{m} \mathrm{y} \pi_{d}$ son las peligrosidades específicas consideradas en la sección anterior. En cuanto a los modificadores se tiene que $\varphi_{a}$ es el factor de modificación para el año, $\varphi_{a m}$ para el mes y $\varphi_{a d}$ para el día, más específicamente si se usa la teoría de procesos estocásticos para calcular las peligrosidades específicas estos factores se pueden calcular simplemente como:

$\varphi_{a}=\frac{\pi_{a}}{\pi_{0}}-1, \varphi_{a m}=\frac{\pi_{a m}}{\pi_{a}}-1, \varphi_{a d}=\frac{\pi_{a d}}{\pi_{a}}-1$

Estos modificadores de accidentalidad pueden ser calculados para cada año y cada situación específica y se presentan en la tabla 2.

\begin{tabular}{|c|c|c|c|c|c|c|c|c|c|}
\hline \multicolumn{10}{|c|}{ Factor de modificación especifica de la peligrosidad especifica según el año } \\
\hline Año & 2007 & 2008 & 2009 & 2010 & 2011 & 2012 & 2013 & 2014 & 2015 \\
\hline$\varphi_{\mathrm{a}}$ & 0,073 & $-0,117$ & $-0,113$ & $-0,108$ & 0,062 & 0,106 & 0,115 & 0,050 & 0,084 \\
\hline \multicolumn{10}{|c|}{ Factor de modificación especifica de la peligrosidad especifica según el mes } \\
\hline$\varphi_{\mathrm{am}}$ & 2007 & 2008 & 2009 & 2010 & 2011 & 2012 & 2013 & 2014 & 2015 \\
\hline Enero & 0,024 & $-0,157$ & $-0,154$ & $-0,149$ & 0,013 & 0,055 & 0,063 & 0,002 & 0,034 \\
\hline Febrero & $-0,009$ & $-0,184$ & -0181 & $-0,176$ & $-0,019$ & 0,022 & 0,030 & $-0,030$ & 0,001 \\
\hline Marzo & 0,036 & $-0,147$ & -0144 & $-0,139$ & 0,025 & 0,068 & 0,076 & 0,014 & 0,046 \\
\hline Abril & 0,066 & $-0,122$ & $-0,119$ & $-0,114$ & 0,055 & 0,099 & 0,108 & 0,044 & 0,077 \\
\hline Mayo & 0,000 & $-0,177$ & $-0,173$ & $-0,169$ & $-0,010$ & 0,031 & 0,039 & $-0,021$ & 0,010 \\
\hline Junio & $-0,002$ & -0179 & $-0,175$ & $-0,171$ & $-0,013$ & 0,029 & 0,037 & $-0,023$ & $-0,008$ \\
\hline Julio & $-0,011$ & $-0,186$ & $-0,183$ & $-0,178$ & $-0,022$ & 0,019 & 0,027 & $-0,032$ & $-0,001$ \\
\hline Agosto & 0,260 & 0,038 & 0,042 & 0,048 & 0,247 & 0,300 & 0,310 & 0,234 & 0,274 \\
\hline Septiembre & 0,082 & $-0,109$ & $-0,106$ & $-0,101$ & 0,071 & 0,116 & 0,124 & 0,060 & 0,093 \\
\hline Octubre & 0,019 & $-0,161$ & $-0,158$ & $-0,153$ & 0,008 & 0,050 & 0,058 & $-0,002$ & 0,029 \\
\hline Noviembre & 0,007 & $-0,171$ & $-0,168$ & $-0,163$ & $-0,003$ & 0,038 & 0,046 & 0,014 & 0,017 \\
\hline Diciembre & 0,058 & $-0,129$ & $-0,125$ & $-0,120$ & 0,047 & 0,091 & 0,100 & 0,036 & 0,069 \\
\hline \multicolumn{10}{|c|}{ Factor de modificación especifica de la peligrosidad especifica según el día } \\
\hline$\varphi_{\mathrm{ad}}$ & 2007 & 2008 & 2009 & 2010 & 2011 & 2012 & 2013 & 2014 & 2015 \\
\hline Lunes & 0,0161 & 0,0502 & 0,0583 & 0,0594 & 0,0905 & 0,0523 & 0,0829 & 0,0161 & 0,0502 \\
\hline Martes & 0,0758 & 0,046 & 0,0958 & 0,0912 & 0,1195 & 0,0713 & 0,0387 & 0,0758 & 0,046 \\
\hline Jueves & 0,0631 & $-0,0139$ & 0,0819 & 0,0622 & 0,065 & 0,1793 & 0,1602 & 0,0631 & $-0,0139$ \\
\hline Viernes & 0,2354 & 0,0809 & 0,1403 & 0,0829 & 0,0708 & 0,173 & 0,0387 & 0,147 & 0,0809 \\
\hline Sábado & $-0,0402$ & $-0,0335$ & $-0,1403$ & $-0,174$ & $-0,1276$ & $-0,2383$ & $-0,1934$ & $-0,0402$ & $-0,0335$ \\
\hline Domingo & $-0,2893$ & $-0,2859$ & $-0,2736$ & $-0,2983$ & $-0,3167$ & $-0,3653$ & $-0,3591$ & $-0,2893$ & $-0,2859$ \\
\hline
\end{tabular}

Tabla 2. Modificadores de macroaccidentalidad específicos, para el día según el año. 


\section{Discusión}

En este artículo se han analizado datos de una base amplia de más de 250 mil accidentes registrados, mediante la teoría de procesos estocásticos. Se ha encontrado que dicha teoría permite entender la distribución de accidentes y explicar a su vez la sobredispersión observada respecto a un modelo de Poisson. Resulta que la distribución binomial aparece porque la población de conductores puede entenderse como una mezcla no homogénea de conductores con diferentes capacidades, otros estudios que se limitaron a buscar un ajuste y encontraron evidencia de la que binomial negativa ajustaban bien los datos no aportan explicación de cuál es la causa subyacente de que dicha distribución parezca funcionar. Además el análisis propuesto permite determinar cómo es la distribución de destrezas efectivas de los conductores (aunque esta puede cambiar según el grado de cansancio, las condiciones atmosféricas y el estado del vehículo y la vía). Resulta que estas destrezas medidas por la peligrosidad intrínseca de cada conductor pueden representarse razonablemente por una distribución gamma de dos parámetros, ver ecuación (8). Esos dos parámetros dan directamente los dos parámetros que dan lugar a la distribución binomial, por lo cual los parámetros de la distribución de accidentes quedan explicados en términos de una distribución más fundamental (la de la distribución de destrezas/peligrosidades intrínsecas de los conductores). Esto constituye una profundización en la comprensión de qué parámetros estadísticos regulan la producción de accidentes.

Otro punto importante que es interesante discutir son las mejoras en los ajustes si en lugar de una distribución binomial negativa pura se usa una distribución binomial con modificación de Lindley (Binomial NegativaLindley). Si bien el ajuste del número de accidentes es mucho mejor, debe notarse que es están usando más parámetros para ajustar los mismos datos, con lo cual es esperable que el ajuste mejore como muchos autores detectan. Nótese que una distribución binomial negativa está caracterizada por dos parámetros $(r, \beta)$ donde $r \in \mathbb{N}$ y $\beta \in \mathbb{R}^{+}$, mientras que una binomial negativa-Lindley viene dada por tres parámetros, dos como los anteriores y uno adicional que da una mezcla de tipo Lindley de binomiales (ver [14]). Sin embargo, ese procedimiento no tiene un fundamento teórico sólido y previsiblemente otras formas de ajustar los datos a una binomial negativa modificada, que contenga más de los dos parámetros asociados a una binomial negativa simple. Además, en el caso de usar una binomial negativa-Lindley no es fácil ver cuál es la distribución que da la distribución de peligrosidades de los conductores, por lo que los tres parámetros ya no son interpretables en términos de algo más simple, además la mezcla para producir sobredispersión se aplica sobre los datos de frecuencias de accidentes y no sobre algo que explique cómo se producen esos accidentes. Por todas estas razones aunque una Binomial Negativa-Lindley puede obtener mejores ajustes en realidad, hace uso de un procedimiento de fuerza bruta aumentando el número de parámetros ajustables, cosa que se podría hacer de muchas maneras diferentes con resultados similares, y teóricamente no mejora la comprensión del proceso estocástico subyacente a la producción de los accidentes. Por esa razón, consideramos que esa no es la vía en la que debería procederse para entender la manera estocástica en que se producen los accidentes.

\section{Conclusiones}

Se ha analizado la macroaccidentalidad de un área red viaria extensa durante casi una década, lo cual incluye más de 250 mil accidentes de diferentes tipos. Se ha encontrado evidencia empírica que bajo circunstancias específicas el número de accidentes en un período de tiempo sigue una distribución binomial negativa y en todos los casos analizados se ve que test $\chi^{2}$ es superado, por lo que el ajuste puede considerarse adecuado. Además se ha propuesto una explicación estocástica, en la que se considera que en todo momento la población de conductores es una mezcla heterogénea (la peligrosidad intrínseca de cada conductor es diferente y la población puede 
representarse mediante una distribución gamma). Esto lleva a que el ritmo de accidentes se producirá según un proceso estocástico de Poisson mixto, en particular un proceso de Pascal-Pólya que da lugar a la menciona distribución binomial negativa. El artículo presenta la originalidad de explicar la razón de la aparición de este tipo de distribución, a diferencia de otros trabajos similares que se limitan a señalar que ese tipo de distribuciones parecen modelizar adecuadamente los datos, sin más explicación.

\section{Referencias}

1 Organización Mundial de la Salud (2015) Global Status Report On Road Safety, p. 2.

2 Dirección General de Tráfico (2017) Anuario estadístico general de 2016, Archivo pdf.

3 Shankar, V., Mannering, F., \& Barfield, W. (1995) "Effect of roadway geometrics and environmental factors on rural accident frequencies." Accident Analysis \& Prevention, 27(3), pp. 371-389.

4 Poch, M., \& Mannering, F. (1996) "Negative binomial analysis of intersection-accident frequencies", Journal of transportation engineering, 122(2), pp. 105-113.

5 Hammerslag, R., Roos, J. P., \& Kwakernaak, M. (1982) "Analysis of accidents in traffic situations by means of multiproportional weighted Poisson model." Transp. Res. Rec. 847, Transp. Res. Board, Washington, D.C., pp. 29-36.

6 Joshua, S. C., \& Garber, N. J. (1990) "Estimating truck accident rate and involvements using linear and Poisson regression models." Transp. Ping. and Technol., 15(1), pp. 41-58.

7 Chin, H. C., \& Quddus, M. A. (2003) "Applying the random effect negative binomial model to examine traffic accident occurrence at signalized intersections." Accident Analysis \& Prevention, 35(2), pp. 253-259.

8 Ladron de Guevara, F., Washington, $S$. P., \& Oh, J. (2004) "Forecasting crashes at the planning level: simultaneous negati- ve binomial crash model applied in Tucson, Arizona." Transportation Research Record, 1897(1), pp. 191-199.

9 Wei, F. \& Lovegrove, G. (2013) "An empirical tool to evaluate the safety of cyclists: Community based, macro-level collision prediction models using negative binomial regression." Accident Analysis \& Prevention, 61, pp. 129-137.

10 Arregui-Dalmases, C., García-Vilana, S., Sánchez-Molina, D., \& Velázquez-Ameijide, J. “Àmbit de la Mobilitat i el trànsit." Informe final de la Càtedra de Seguretat Viària de Motocicletes del Servei Català del Trànsit, 2017.

11 Mikosch, Thomas (2013) Non-life insurance mathematics: an introduction with the Poisson process, Springer Science \& Business Media. ISBN 978-3-540-88233-6.

12 Lord, D. \& Geedipally, S. R. (2011) "The negative binomial-Lindley distribution as a tool for analyzing crash data characterized by a large amount of zeros." Accident Analysis \& Prevention, 43(5), pp. 1738-1742.

13 Geedipally, S. R., Lord, D. \& Dhavala, S. S. (2012) "The negative binomial-Lindley generalized linear model: Characteristics and application using crash data." Accident Analysis \& Prevention, 45, pp. 258-265.

14 Cakmakyapan, S. \& Ozel, G. (2016) "The Lindley family of distributions: properties and applications." Hacettepe Journal of Mathematics and Statistics, 46(6), pp. 11131137.

15 Teugels, J. L \& Vynckier, P. (1996) "The structure distribution in a mixed Poisson process." International Journal of Stochastic Analysis, 9(4), pp. 489-496.

16 Konno, H. (2010): "On the exact solution of a generalized Pólya process." Advances in Mathematical Physics. Article ID 504267 doi:10.1155/2010/504267.

17 Badía, F.G., Sangüesa C., \& Cha, J. H. (2018) "Univariate and multivariate stochastic comparisons and ageing properties of the generalized Pólya process." Journal of Applied Probability, 55(1), pp. 233-253. 


\title{
Electrodeposición de recubrimientos de fosfato de calcio sobre titanio
}

\author{
E. Vidal ${ }^{1,2}$, E. Rupérez ${ }^{1,2}$, D. Rodríguez ${ }^{1,2}$ \\ I Biomaterials, Biomechanics and Tissue Engineering Group, Department of Materials Science and \\ Metallurgical Engineering, Universitat Politècnica de Catalunya (UPC) \\ 2 Center for Research in nanoEngineering, UPC
}

\begin{abstract}
Resumen
El titanio y sus aleaciones son biomateriales usados frecuentemente en implantes y prótesis. Sin embargo, estos biomateriales metálicos tienen limitaciones relacionadas con la bioactividad.

Una vía para aumentar la bioactividad del titanio es añadir un recubrimiento de fosfato de calcio (CaP) a la superficie mediante deposición electroquímica. Esta técnica tiene como ventajas la baja temperatura del proceso y un buen control del espesor y la composición química de la capa depositada. Durante el proceso, sin embargo, se producen burbujas de hidrógeno en las proximidades del cátodo, que dificultan la obtención de capas uniformes.

El presente estudio propone usar corriente pulsada para la electrodeposición como mecanismo para reducir la producción de burbujas de hidrógeno y mejorar el equilibrio de la concentración de iones en la solución, permitiendo la formación de capas de fosfato de calcio más uniformes.
\end{abstract}

Palabras clave: Titanio, electrodeposición, fosfato de calcio

\begin{abstract}
Titanium and its alloys are biomaterials commonly used in implants and prostheses. However, these metallic biomaterials have limited bioactivity.

One way to increase the bioactivity of titanium is to add a coating of calcium phosphate (CaP) to the surface by electrochemical deposition. This technique has as advantages the low temperature of the process and a good control of the thickness and chemical composition of the deposited layer. During the process, however, hydrogen bubbles may occur near the cathode, which makes it difficult to obtain uniform layers.

The present study proposes using pulsed current for electrodeposition as a mechanism to reduce the production of hydrogen bubbles and improve the equilibrium of the concentration of ions in the solution, allowing the formation of more uniform layers of calcium phosphate.
\end{abstract}

Keywords: Titanium, electrodeposition, calcium phosphate

Correspondencia:

Elia Vidal.

E-mail: elia.vidal@upc.edu

DOI: $10.5821 /$ sibb.26.1.8768 


\section{Introducción}

El titanio es un biomaterial con muy buena biocompatibilidad, en parte debida a su elevada resistencia a la corrosión en el medio fisiológico. El titanio presenta una buena resistencia a la corrosión debido a la gran reactividad que tiene con el oxígeno formando una capa de pasivación estable. La reactividad del titanio no da lugar a productos tóxicos, por lo que es ampliamente utilizado en aplicaciones biomédicas. Aunque biocompatible, el titanio no es bioactivo, lo que significa que no es capaz de inducir una actividad biológica específica [1].

Existen diversas alternativas para mejorar la bioactividad de un biomaterial metálico como el titanio, como por ejemplo tratamientos químicos que formen titanato en la superficie. Otros procesos se basan en la formación de un recubrimiento bioactivo (normalmente de fosfato de calcio) sobre el titanio, por proyección (plasma-spray), deposición sol-gel, o mediante laser-cladding, entre otras posibilidades. Entre estos procesos de recubrimiento, la deposición por pulverización de plasma es la única técnica utilizada comercialmente para recubrir prótesis, pero presenta ciertos inconvenientes, como la falta de uniformidad, el control limitado de la composición de la capa y la posible delaminación, debido a la altísima temperatura de procesado [2].

Otra vía para añadir un recubrimiento de fosfato de calcio a la superficie del titanio es mediante deposición electroquímica. Esta técnica tiene como ventajas la baja temperatura del proceso y un buen control del espesor y la composición química de la capa depositada. Durante el proceso, sin embargo, se producen burbujas de hidrógeno en las proximidades del cátodo, que dificultan la obtención de capas uniformes [3].

El presente estudio propone usar corriente pulsada para la electrodeposición como mecanismo para reducir la producción de burbujas de hidrógeno y mejorar la uniformidad de la concentración de iones en la solución, permitiendo la formación de capas de fosfato de calcio más uniformes.

\section{Materiales y Métodos}

Se prepararon muestras de titanio puro grado 2 de $10 \mathrm{~mm}$ de diámetro y $2 \mathrm{~mm}$ de espesor a partir de barras de titanio del mismo diámetro (Zapp Precision Metals GmbH, Alemania). Los discos fueron desbastados con discos de pulir de carburo de silicio desde 320 hasta 4000 grit (Buehler, EEUU) y finalmente pulidos con acabado espejo mediante sílice coloidal de 0,05um de diámetro.

Las muestras pulidas se limpiaron en un baño de ultrasonidos con soluciones de acetona, etanol y agua ultrapura. Antes de la electrodeposición, las muestras se trataron en una solución de $\mathrm{NaOH} 5 \mathrm{M}$ (Sigma Aldrich, EEUU) durante 24 horas a $60^{\circ} \mathrm{C}$, se enjuagaron con agua ultrapura y se secaron, almacenándose en un desecador.

La electrodeposición se realizó sobre las muestras de titanio con un equipo Parstat 2273 (Princeton Applied Research, EEUU) en régimen catódico. Se utilizó un electrodo de platino como ánodo. El electrolito se preparó mezclando $0,04 \mathrm{M} \mathrm{Ca}\left(\mathrm{NO}_{3}\right)_{2} \cdot 4 \mathrm{H}_{2} \mathrm{O}$ y $0,02 \mathrm{M}$ $\mathrm{NH}_{4} \mathrm{H}_{2} \mathrm{PO}_{4}$ (Sigma Aldrich). Las muestras se procesaron en el electrolito indicado durante 30 minutos a una temperatura de $40^{\circ} \mathrm{C}$ y una densidad de corriente de $2 \mathrm{~mA} / \mathrm{cm}^{2}$. Se compararon dos condiciones de corriente en el tratamiento (no pulsada / pulsada con pulso unipolar o bipolar, 300 ciclos de 4 segundos, figura 1).

Las propiedades fisicoquímicas del recubrimiento se evaluaron mediante diversas técnicas. La morfología y química superficial se estudió con microscopía electrónica de barrido complementada con sonda EDS (SEM Carl Zeiss NTS GmbH, Alemania). La microestructura del fosfato de calcio del recubrimiento fue analizada mediante difracción de rayos $\mathrm{X}(\mathrm{DRX})$ utilizando una radiación de $\mathrm{K} \alpha$ de $\mathrm{Cu}$ monocromática (Bruker D8 Advance Instrument, Alemania) a una velocidad de escaneo de $1 \%$, en el rango $2 \theta$ de 4 a $45^{\circ}$. La rugosidad del recubrimiento fue caracterizada con un equipo de interferometría de luz blanca WYKO NT9300 (Veeco Instruments, EEUU). 


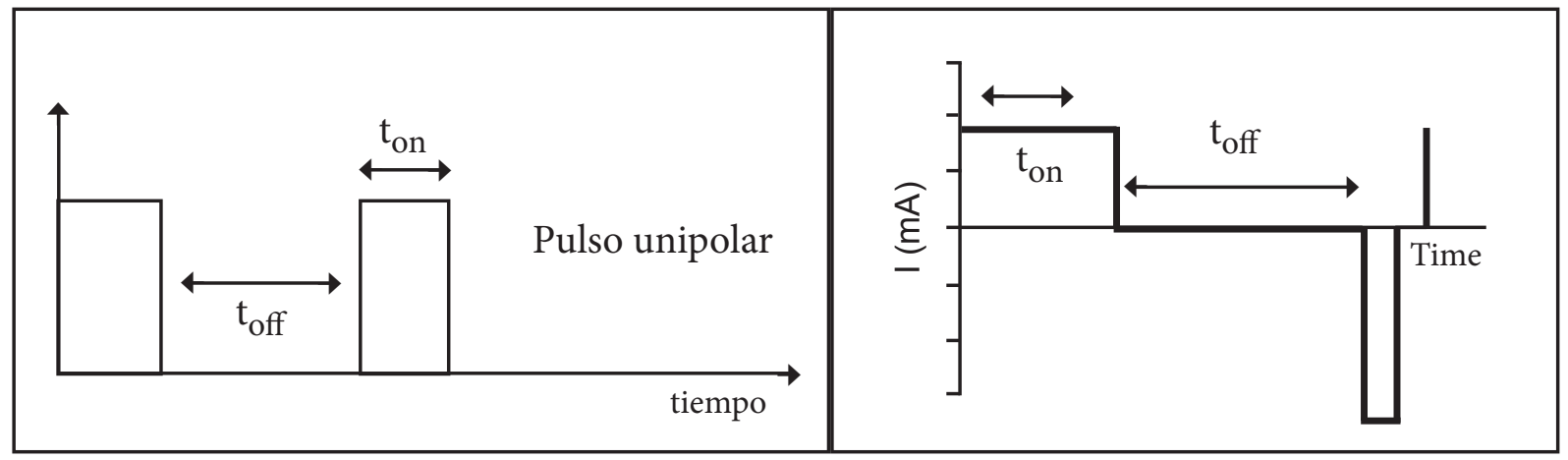

Figura 1. Esquema de electrodeposición en pulso unipolar (izquierda) y bipolar (derecha).

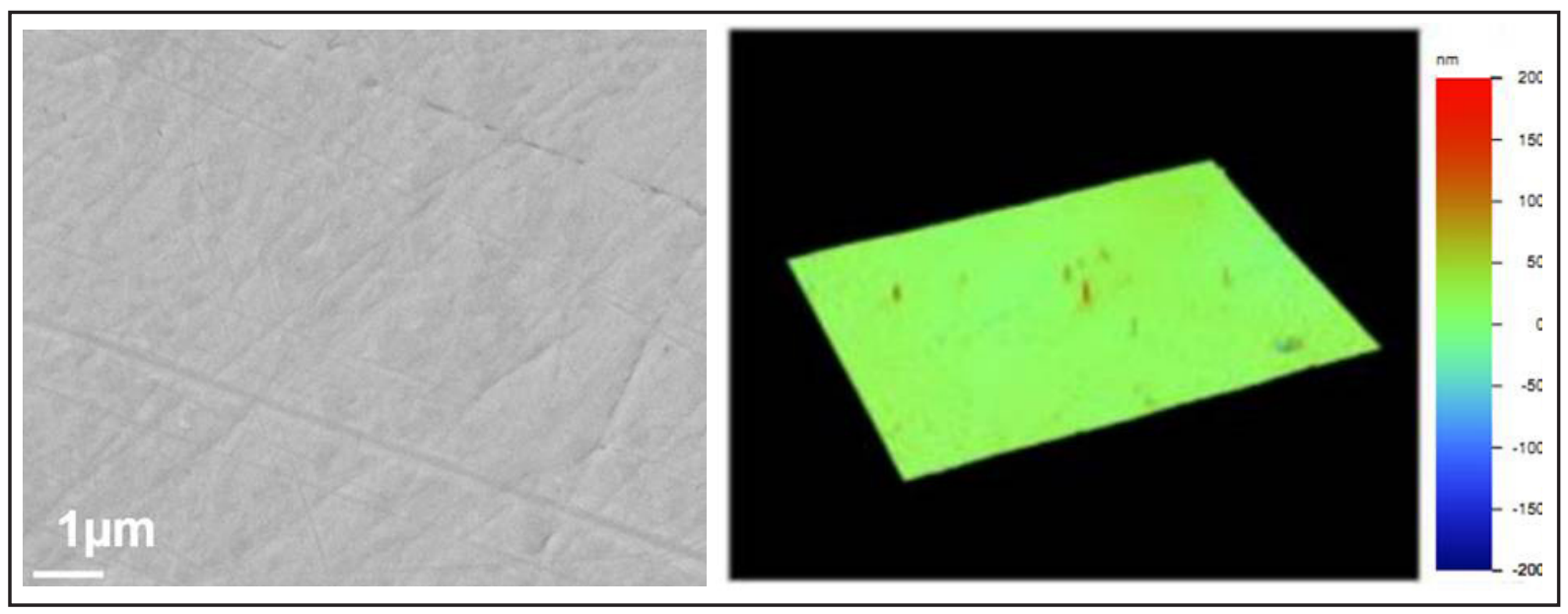

Figura 2. Imagen de microscopía electrónica de una muestra de titanio pulida, y reconstrucción de la topografía superficial mediante interferometría de luz blanca.
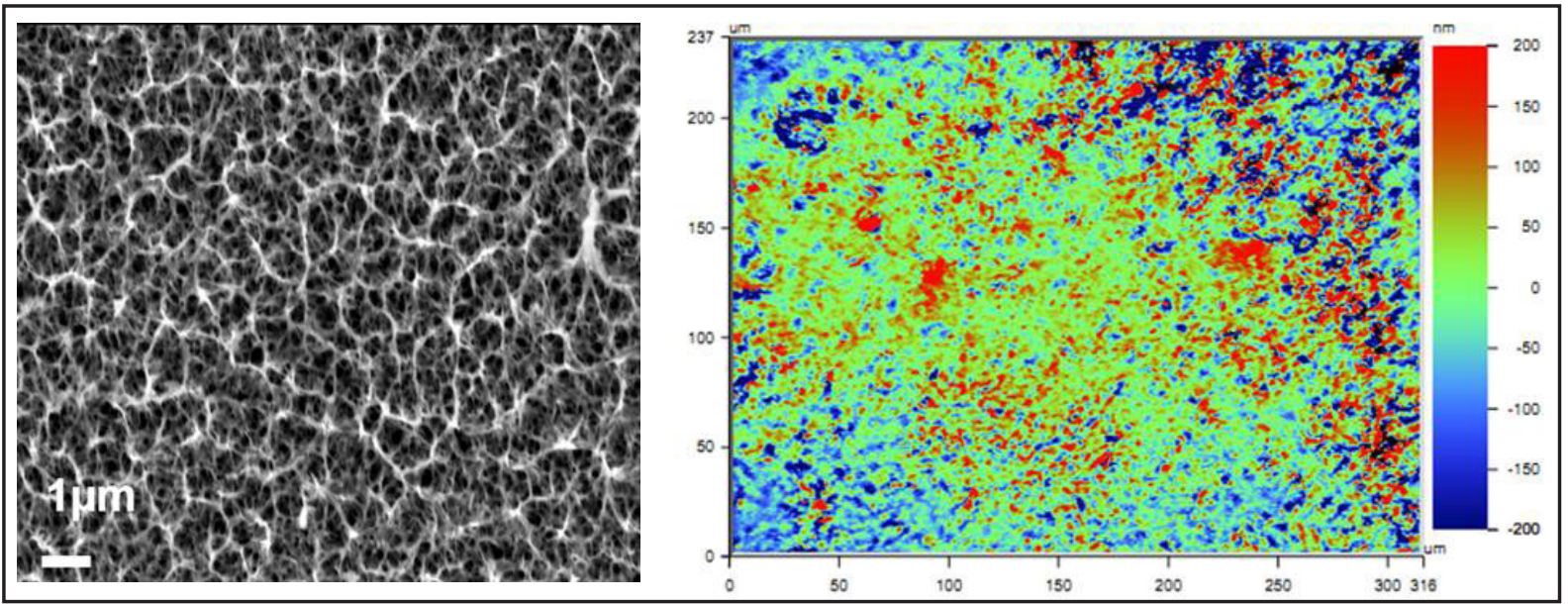

Figura 3. Imagen de microscopía electrónica de una muestra de titanio tratada con $\mathrm{NaOH}$, y reconstrucción de la topografía superficial mediante interferometría de luz blanca. 


\section{Resultados y Discusión}

Las muestras pulidas de titanio presentaron un acabado muy liso, como muestra la imagen de microscopio electrónico en la figura 2. Las medidas de rugosidad superficial con interferometría de luz blanca dieron una rugosidad media Ra de 12,6nm. Este acabado superficial permite evaluar los efectos de los tratamientos posteriores sin interferencias debidas al estado superficial previo.

Las muestras de titanio tratadas con una solución de $\mathrm{NaOH} 5 \mathrm{M}$ mostraron una superficie atacada con pérdida del pulido superficial (Figura 3). La rugosidad superficial Ra de las muestras tras el tratamiento es de 74,6nm. Esta evolución en la morfología superficial se debe al efecto corrosivo del $\mathrm{NaOH}$ en el titanio a temperaturas elevadas, y es empleado en algunas aplicaciones para incrementar la rugosidad superficial del titanio para aplicaciones biomédicas [4].

Tras el tratamiento electroquímico con corriente continua a $2 \mathrm{~mA} / \mathrm{cm}^{2}$, las muestras mostraban en la superficie el efecto de las burbujas de hidrógeno en el recubrimiento de fosfato de calcio formado sobre el titanio (figura 4) $[5]$.

La presencia de los defectos debidos a las burbujas de hidrógeno incrementa la rugosidad

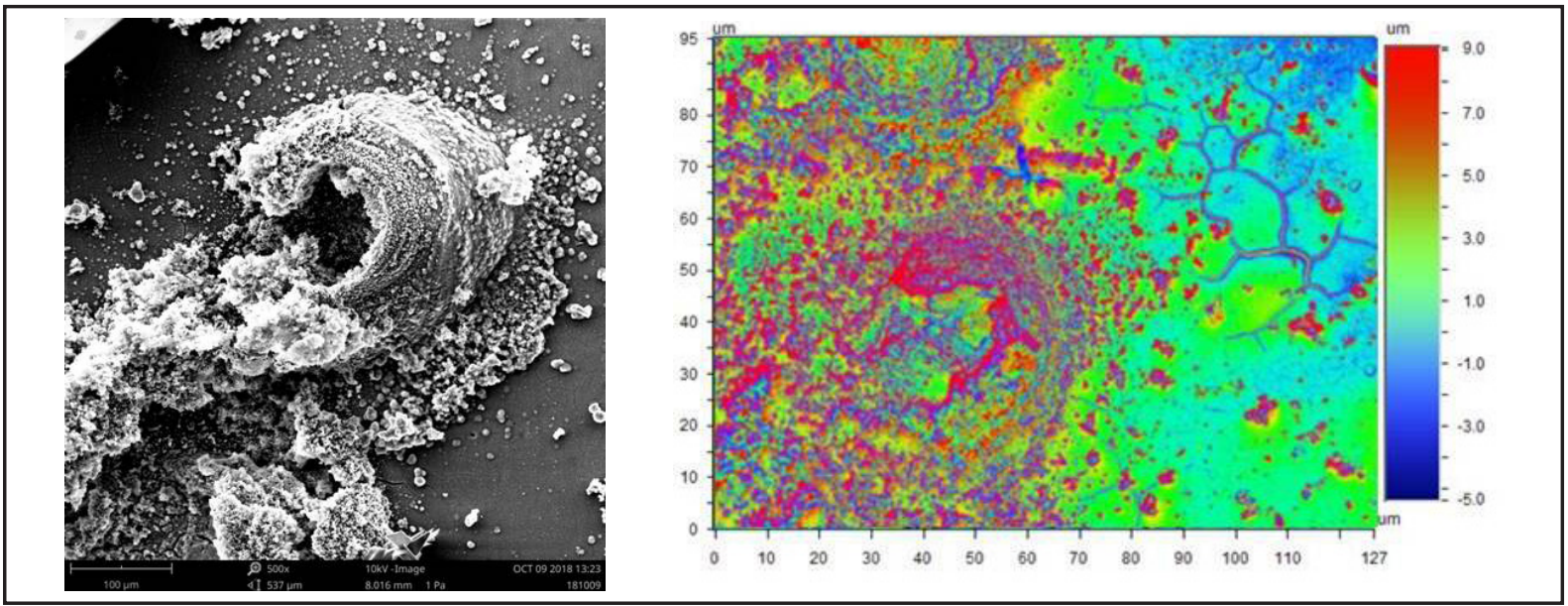

Figura 4. Imagen de microscopía electrónica de una muestra de titanio tras un tratamiento con corriente continua, y reconstrucción de la topografía superficial mediante interferometría de luz blanca.

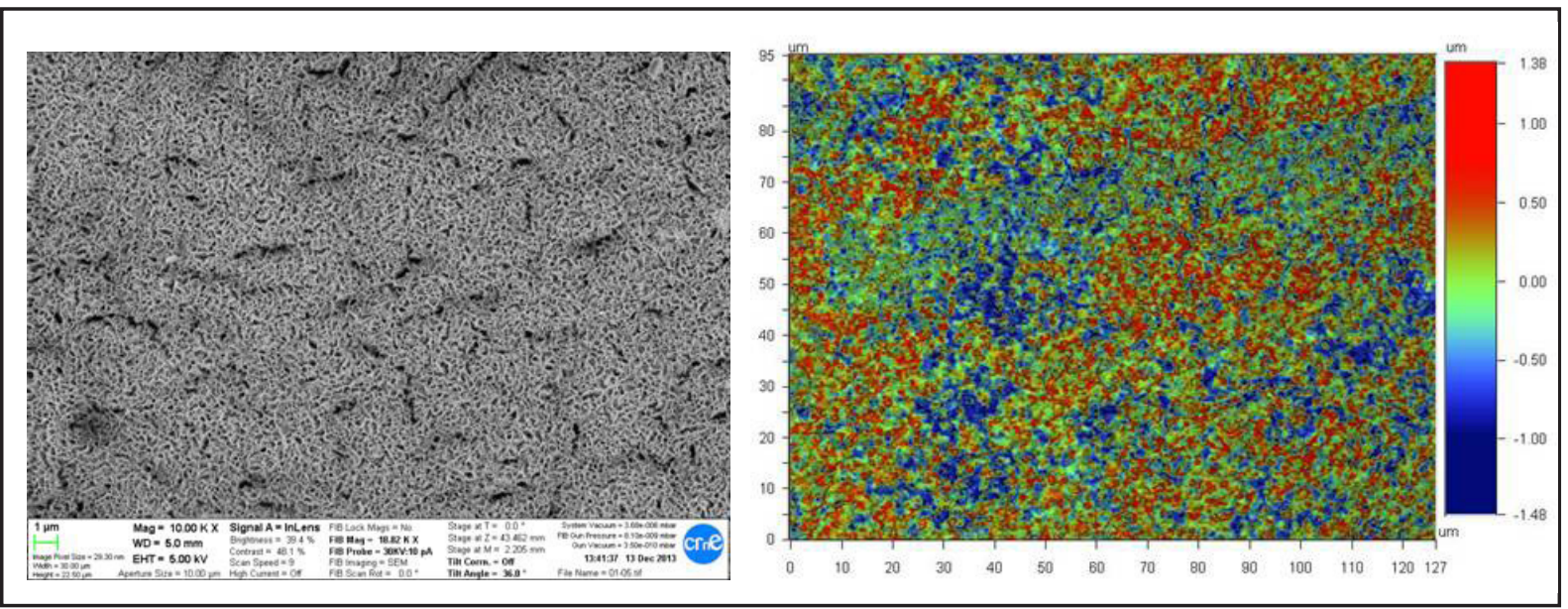

Figura 5. Imagen de microscopía electrónica de una muestra de titanio tras un tratamiento unipolar, y reconstrucción de la topografía superficial mediante interferometría de luz blanca. 
superficial a $1,80 \mu \mathrm{m}$. El valor de rugosidad incrementado no es un problema para la aplicación de este tratamiento, pero la presencia de los defectos debidos a las burbujas debilita la integridad estructural de la capa y hace más probable que se separe del sustrato [6]

En el caso de las muestras tratadas en régimen unipolar, las superficies presentaban una morfología compleja, con poros en la superficie (figura 5). Con este tratamiento, sin embargo, no se detectaron estructuras circulares como las que se habían observado en las muestras tratadas con corriente continua. La rugosidad superficial es de $0,32 \mu \mathrm{m}$, un valor bajo, como era de esperar al observar la morfología en microscopía electrónica.
Al examinar las superficies tratadas con más detalle, se observó la presencia de una morfología de tipo placas, con presencia de defectos en la continuidad de la capa (figura 6).

En el caso de los tratamientos electroquímicos en régimen bipolar, las superficies tratadas también presentaban superficies de morfología compleja, pero a diferencia de las muestras tratadas en régimen unipolar no se observaron poros o defectos en la superficie (figura 7). La rugosidad superficial es de 1,56 $\mu \mathrm{m}$, mucho mayor que la rugosidad en las muestras tratadas en régimen unipolar.

Al examinar la superficie tratada en régimen bipolar al microscopio electrónico con mayor resolución, se observó una morfolo-

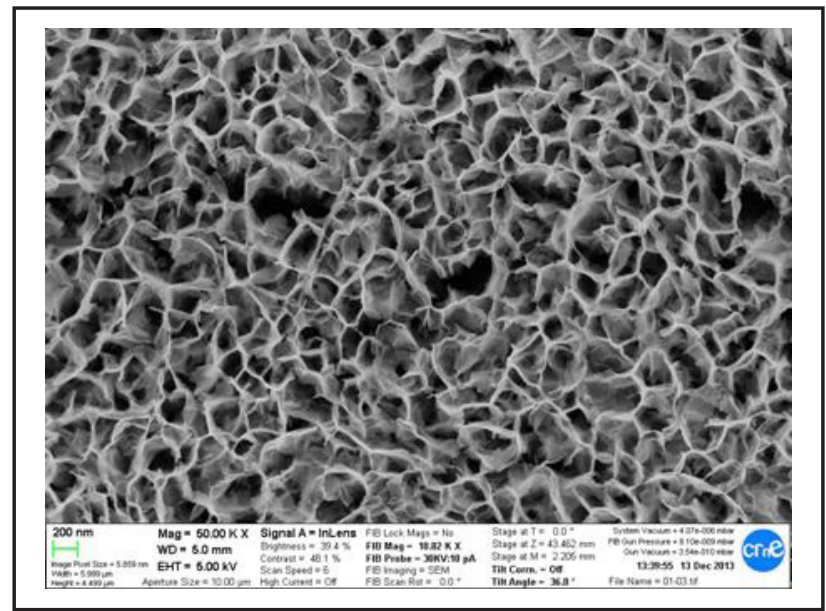

Figura 6. Detalle de la superficie de una muestra de titanio tras un tratamiento electroquímico unipolar.

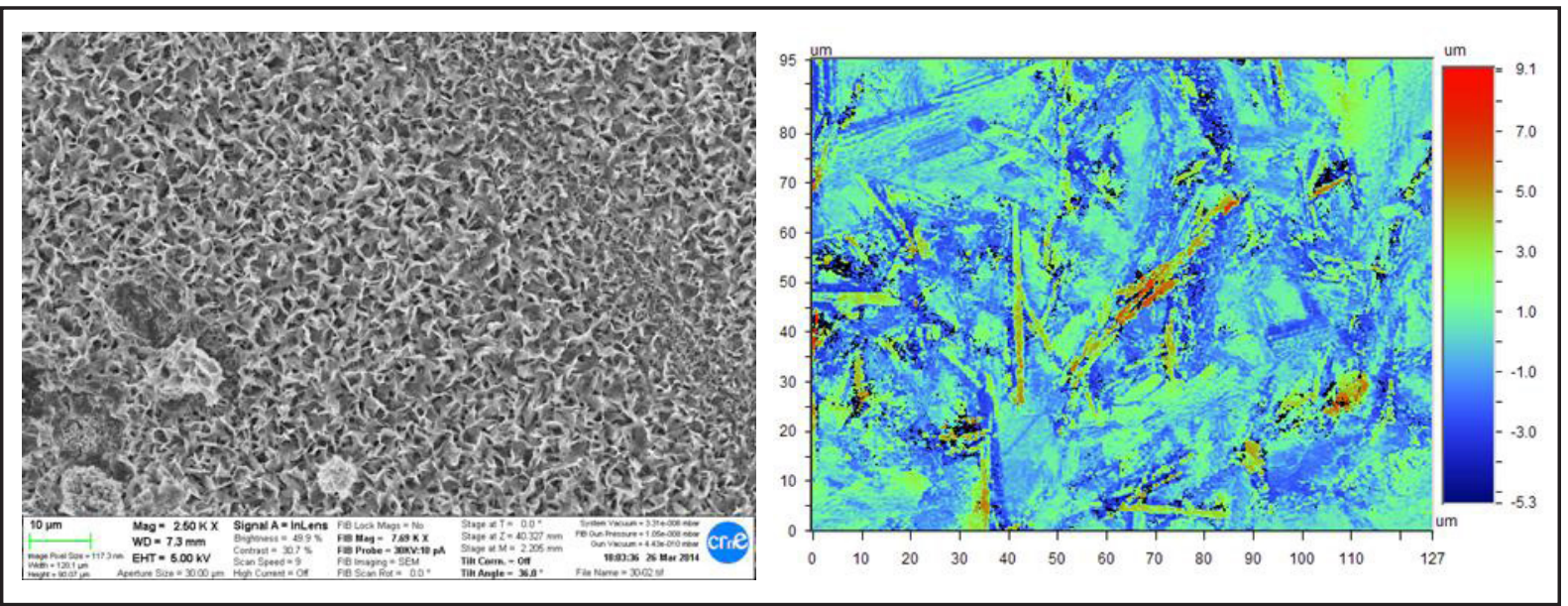

Figura 7. Imagen de microscopía electrónica de una muestra de titanio tras un tratamiento con corriente bipolar, y reconstrucción de la topografía superficial mediante interferometría de luz blanca. 
gía de placas similar a la obtenida en régimen unipolar, pero mucho más homogénea, y con mayores tamaños de placas (Figura 8).

Las observaciones en microscopía electrónica permiten comprobar que el uso de corriente pulsada permite obtener capas de fosfato de calcio más uniformes. La interrupción de la corriente no permite la formación de grandes burbujas, pero como se observa en las muestras tratadas en régimen unipolar, no desaloja las burbujas de la superficie, generando defectos. Sólo las muestras tratadas con un régimen bipolar consiguen desalojar las burbujas de la superficie, facilitando la formación de la capa de fosfato de calcio en la superficie de titanio al no interferir las burbujas de hidrogeno [7].

El análisis de las muestras tratadas con corriente pulsada mediante difracción de rayos $\mathrm{X}$ muestra en el espectro obtenido picos cuya indexación corresponde la presencia de fases cristalinas de fosfato de calcio (figura 9).

En el caso de las muestras tratadas con corriente unipolar, se detectaron picos cuya indexación corresponde a la hidroxiapatita, brushita y fosfato octacálcico (OCP). Esto puede ser debido a un aumento de la concentración de iones hidroxilo en el electrolito que promueve la precipitación de OCP. Estas fases de fosfato de calcio podrían influir en el cre-

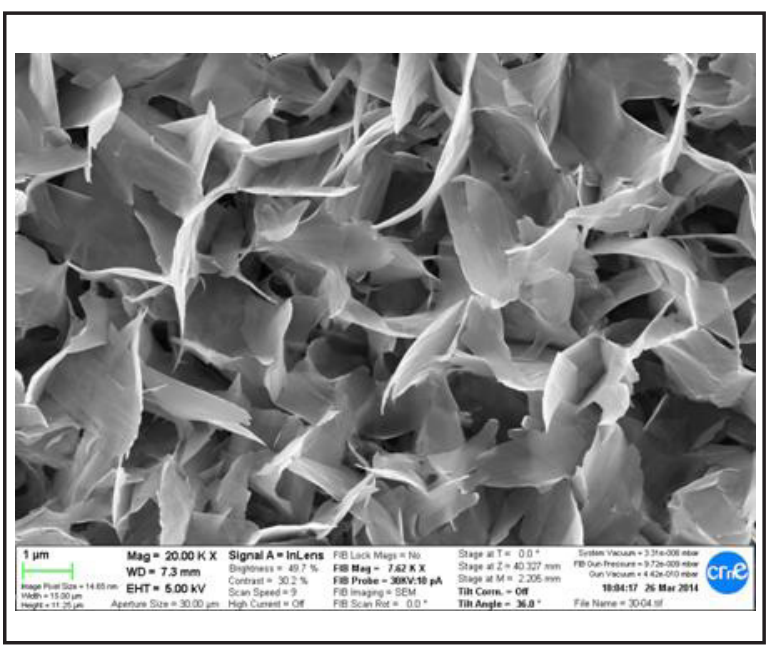

cimiento óseo, ya que se conocen como precursores de la formación de HA. El espectro de DRX de las muestras tratadas en régimen bipolar muestra picos de brushita y OCP, pero no de hidroxiapatita (figura 9).

En ambos casos, el espectro muestra una señal poco intensa de titanio metálico fase $\alpha$, hecho que sugiere que la capa de fosfato de calcio tiene un espesor micrométrico, estimado en 5-10 $\mu \mathrm{m}$.

\section{Conclusiones}

Se crearon con éxito recubrimientos de fosfato de calcio en la superficie de muestras de titanio mediante un proceso electroquímico. Se ha conseguido mejorar la calidad de la capa obtenida ajustando el pulsado de la corriente aplicada al sistema para reducir la formación de burbujas en la superficie a tratar.

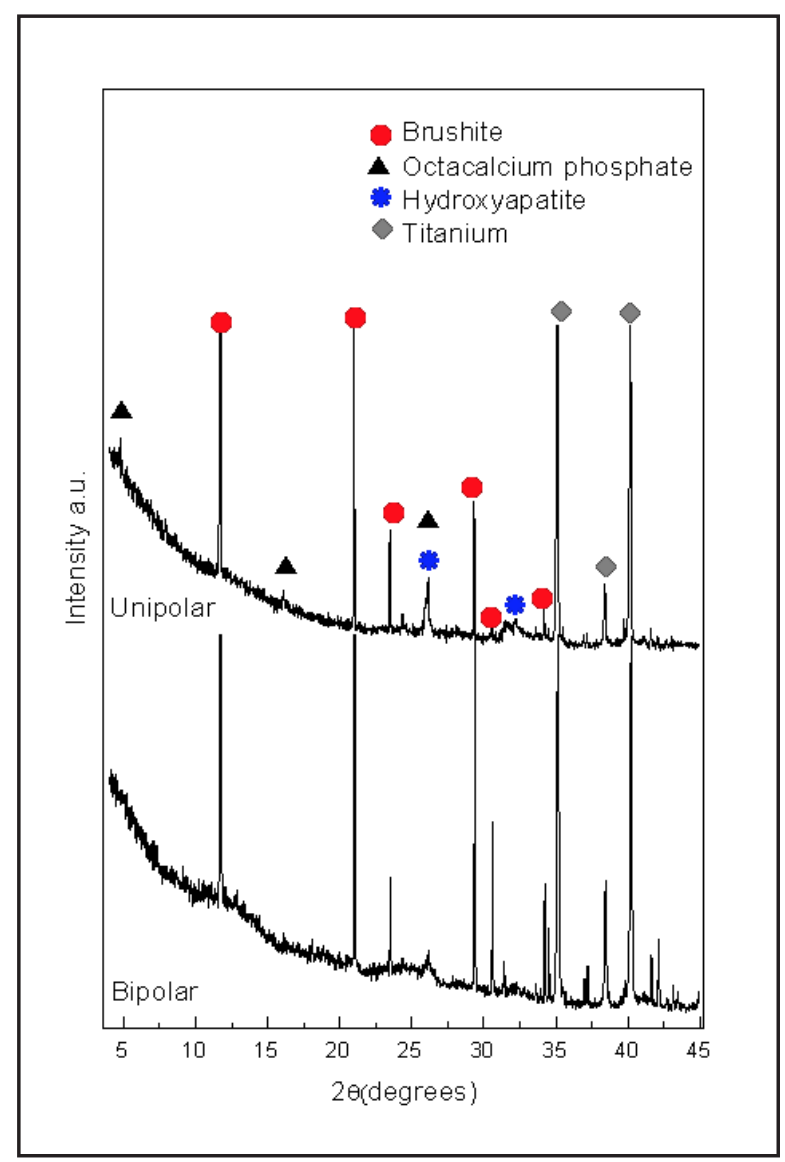

Figura 8. (Izquierda) Detalle de la superficie de una muestra de titanio tras un tratamiento electroquímico bipolar.

Figura 9. (Derecha) Espectro de DRX de una muestra de titanio tras un tratamiento electroquímico pulsado. 


\section{Agradecimientos}

El presente estudio fue apoyado por la Agencia Catalana d'Administració de Beques Universitàries $\mathrm{i}$ de Recerca (AGAUR FI-DRG). Los autores reconocen al Gobierno español para el apoyo financiero a través del proyecto MAT2015-67183-R, co-financiado por la Unión Europea a través de los Fondos Europeos de Desarrollo Regional.

\section{Referencias}

1 Scharnweber $D$, Flössel $M$, Born $R$, Worch H. Adjusting the chlorhexidine content of calcium phosphate coatings by electrochemically assisted co-deposition from aqueous solutions. J Mater Sci Mater Med 2007;18:391-397.

2 García-Sanz FJ, Mayor MB, Arias JL, Pou J, León B, Pérez-Amor M. Hydroxyapatite coatings: a comparative study between plasma-spray and pulsed laser deposition techniques, J Mater Sci Mater Med 1997;8:861-865.
3 Djošić MS, Panić V, Stojanović J, Mitrić M, Miskovic-Stankovic VB. The effect of applied current density on the surface morphology of deposited calcium phosphate coatings on titanium, Colloids Surf. A Physicochem Eng Asp. 2012;400:36-43.

4 Fujibayashi S, Neo M, Kim HM, Kokubo T, Nakamura T. Osteoinduction of porous bioactive titanium metal. Biomaterials. 2004;25(3):443-450.

5 Vidal E, Buxadera-Palomero J, Pierre C, Manero JM, Ginebra MP, Cazalbou S, Combes C, Rupérez E, Rodríguez D. Single-step pulsed electrodeposition of calcium phosphate coatings on titanium for drug delivery. Surf Coat Techol. 2019;358:266-275.

6 Gopi D, Indira J, Kavitha L. A comparative study on the direct and pulsed current electrodeposition of hydroxyapatite coatings on surgical grade stainless steel, Surf Coat Technol. 2012;206:2859-2869.

7 Chandrasekar MS, Pushpavanam M. Pulse and pulse reverse plating - conceptual, advantages and applications. Electrochim Acta. 2008;53:3313-3322. 


\section{CARTA DEL DIRECTOR SALIENTE}

Estimados lectores de Biomecánica:

Hace 10 años la Sociedad Ibérica de Biomecánica y Biomateriales (SIBB) me propuso como Director de la revista Biomecánica, el Órgano oficial de comunicación de la Sociedad. En aquel entonces tenía una experiencia limitada en la edición de revistas, y me intimidaba tomar el relevo de Conrado Aparicio, el anterior Director, que dejaba el cargo tras haber dirigido la revista con gran éxito. Afortunadamente, su soporte y el de la Junta de la SIBB me permitieron sortear la novedad y dificultades iniciales.

En este periodo de tiempo como Director la revista ha pasado por cambios importantes. El objetivo se sigue manteniendo: ser un mecanismo de comunicación de los miembros de la SIBB y de otros investigadores en los ámbitos de interés de la Sociedad. Ha cambiado, sin embargo, el formato y el acceso a la misma. Desde el año 2011 Biomecánica se publica anualmente en formato digital, y en un movimiento que se avanzaba a los tiempos y con el soporte de la SIBB se decidió que fuera de acceso abierto (Open Access) sin coste para el autor o el lector. Esto es posible gracias al soporte de la SIBB, que sufraga las tareas de maquetación, y también al soporte de la Universitat Politècnica de Catalunya (UPC), que a través de la Iniciativa Digital Politècnica del Servicio de Bibliotecas se encargó de la digitalización de los ejemplares antiguos de la revista y de alojarla en su repositorio digital. Este cambio a formato digital ha permitido ampliar de forma muy notable el alcance de la revista, que actualmente supera las 100.000 descargas de artículos anuales (fuente: https://revistes.upc.edu/index.php/SIBB/statisticsUPC), siendo la revista más consultada del repositorio UPC.

Mi tarea como Director no hubiera sido posible sin el soporte de la SIBB y de todos sus miembros. He de reconocer especialmente la colaboración del Comité Científico de la revista, que supervisa y valora la calidad científica de los manuscritos presentados aportando comentarios para su mejora, del Secretario de Redacción, el Sr. Pere Puig, a quien agradezco su trabajo y colaboración durante estos años, y de la Sra. Roser Alonso, del Servei de Biblioteques de la UPC, que actualiza la revista en el repositorio digital con cada nuevo ejemplar. Finalmente, he de agradecer a todos los autores que han enviado manuscritos para su publicación, y a los lectores de la revista por el tiempo que dedican a la lectura de los mismos.

Tras este tiempo el trabajo como Director sigue siendo apasionante, pero también estoy convencido de que los cambios son positivos, por lo que ha llegado el momento de dar un paso al lado y que otra persona con nuevas ideas tome el relevo de la Dirección de la revista. Agradezco sinceramente al Dr. Diego Velasco Bayón, profesor de la Universidad Carlos III y organizador del XLI Congreso de la SIBB, de que haya aceptado la propuesta de la SIBB para ser el nuevo Director de Biomecánica. Estoy convencido de que dirigirá la revista con gran éxito.

Gracias por su confianza todos estos años.

Un afectuoso saludo,

Daniel Rodríguez Rius 


\section{CARTA DEL DIRECTOR}

Estimados lectores de la revista Biomecánica:

Como nuevo Director de la revista, me gustaría, en primer lugar, destacar a Daniel Rodríguez y su equipo por su trayectoria de tantos años ayudando a que la Biomecánica Deportiva, la Biomedicina y los Biomateriales tengan un foro de debate científico propio en este país a través de los Congresos que se celebran anualmente y especialmente a través de la revista Biomécanica. En segundo lugar, me gustaría agradecer a la Sociedad Ibérica de Biomecánica y Biomateriales (SIBB) la confianza que deposita en mí y en mi equipo.

Como bien sabéis, la SIBB tiene como objetivo de agrupar a cirujanos, médicos, ingenieros, físicos, químicos y especialistas en educación física; en un foro para el intercambio de ideas y al mismo tiempo como estímulo para la investigación.

Un elemento fundamental para lograr dicho objetivo es la revista "Biomecánica", originalmente publicada en papel y actualmente en formato digital "open access", sin cargos para el autor del artículo o el lector del mismo. En la revista se publican revisiones de un tema específico, trabajos originales, resúmenes de Tesis Doctorales, informaciones sobre Congresos y actividades, etc. dentro de los tres ámbitos de la SIBB:

- Área de cirugía ortopédica y traumatología

- Área de biomecánica deportiva

- Área de biomateriales

Espero estar a la altura de las circunstancias y os animo a que participéis activamente en la revista.

Recibid un cordial saludo,

Diego Velasco Bayón 


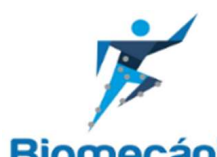

Biomecánica

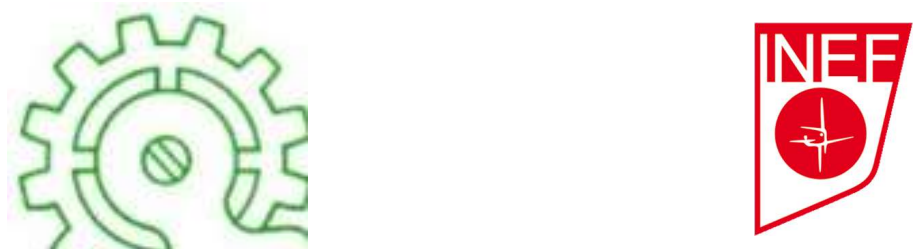

\section{CONGRESO DE LA SOCIEDAD IBÉRICA DE BIOMECÁNICA Y BIOMATERIALES}

\section{5 y 16 de noviembre de 2019}

\section{Facultad de Ciencias de la Actividad Física y del Deporte INEF UNIVERSIDAD POLITECNICA DE MADRID}

\begin{tabular}{|c|c|c|}
\hline & \multicolumn{2}{|c|}{15 de Noviembre } \\
\hline $8.00-9.00$ & \multicolumn{2}{|c|}{ Acreditación } \\
\hline $9.00-9.30$ & \multicolumn{2}{|c|}{ Inauguración } \\
\hline $9.30-11.30$ & $\begin{array}{l}\text { Lesiones músculo esqueléticas del } \\
\text { miembro inferior y columna }\end{array}$ & $\begin{array}{l}\text { Workshop Biomateriales } \\
\text { Obtención y caracterización de la seda } \\
\text { de araña. }\end{array}$ \\
\hline $11.30-12.00$ & \multicolumn{2}{|c|}{ Descanso (Café) } \\
\hline $12.00-14.00$ & $\begin{array}{l}\text { Workshop Biomecánica y } \\
\text { Biomedicina } \\
\text { Análisis de la pisada mediante } \\
\text { sistemas de captura del movimiento } \\
\text { 3D }\end{array}$ & $\begin{array}{l}\text { Mesa Biomateriales } \\
\text { Por determinar }\end{array}$ \\
\hline $14.00-15.00$ & \multicolumn{2}{|c|}{ Comida } \\
\hline $15.00-16.00$ & \multicolumn{2}{|c|}{$\begin{array}{l}\text { Grupos de Investigación de la Universidad Politécnica de Madrid. } \\
\text { Presentación de trabajos }\end{array}$} \\
\hline $16.00-20.00$ & $\begin{array}{l}\text { Comunicaciones y Posters } \\
\text { Biomecánica y Biomedicina }\end{array}$ & $\begin{array}{l}\text { Comunicaciones y Posters } \\
\text { Biomateriales }\end{array}$ \\
\hline 21.00 & \multicolumn{2}{|c|}{ Cena. Entrega Premios } \\
\hline
\end{tabular}

\begin{tabular}{|l|l|l|}
\hline $9.00-11.00$ & $\begin{array}{l}\text { Mesa Biomecánica y Biomedicina } \\
\text { Lesiones músculo esqueléticas del } \\
\text { miembro superior y columna }\end{array}$ & $\begin{array}{l}\text { Mesa Biomateriales } \\
\text { Por determinar }\end{array}$ \\
\hline $11.00-11.30$ & \multicolumn{2}{|c|}{ Presentación Posters (Café) } \\
\hline $11.30-13.00$ & $\begin{array}{l}\text { Presentación MovLab } \\
\text { (Universidad Lusufona de Lisboa) }\end{array}$ & $\begin{array}{l}\text { Workshop Biomateriales } \\
\text { Por determinar }\end{array}$ \\
\hline $13.00-15.00$ & \multicolumn{2}{|c|}{ ASAMBLEA SIBB } \\
\hline
\end{tabular}


